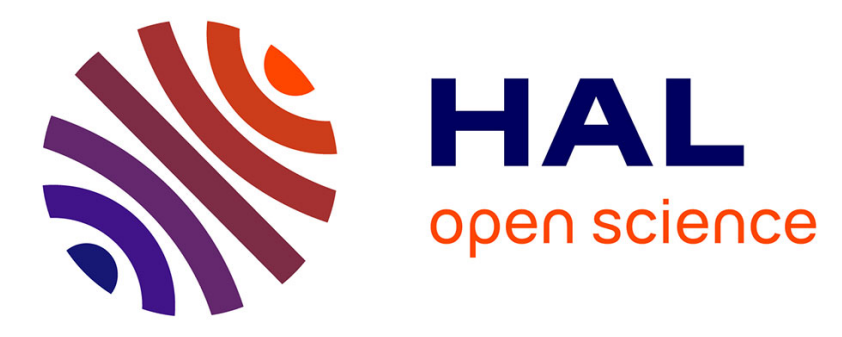

\title{
Structural analysis by bond graph approach: Duality between causal and bicausal procedure
}

Mariem El Feki, Audrey Jardin, Wilfrid Marquis-Favre, Laurent Krähenbühl, Daniel Thomasset

\section{- To cite this version:}

Mariem El Feki, Audrey Jardin, Wilfrid Marquis-Favre, Laurent Krähenbühl, Daniel Thomasset. Structural analysis by bond graph approach: Duality between causal and bicausal procedure. Proceedings of the Institution of Mechanical Engineers, Part I: Journal of Systems and Control Engineering, 2012, 226 (1), pp.82-100. 10.1177/0959651811407708 . hal-00639588

\section{HAL Id: hal-00639588 https://hal.science/hal-00639588}

Submitted on 28 Mar 2019

HAL is a multi-disciplinary open access archive for the deposit and dissemination of scientific research documents, whether they are published or not. The documents may come from teaching and research institutions in France or abroad, or from public or private research centers.
L'archive ouverte pluridisciplinaire HAL, est destinée au dépôt et à la diffusion de documents scientifiques de niveau recherche, publiés ou non, émanant des établissements d'enseignement et de recherche français ou étrangers, des laboratoires publics ou privés. 


\title{
Structural analysis by bond graph approach: Duality between causal and bicausal procedures
}

\author{
Mariem El Feki, Audrey Jardin, Wilfrid Marquis-Favre, Laurent Krähenbühl and Daniel Thomasset
}

\begin{abstract}
The infinite structure of linear time-invariant systems has been principally used to solve control problems. Nevertheless, this system characterization appears interesting in the design and sizing of mechatronic systems as well. Indeed, based on the bond graph language and inverse modelling, a methodology has already been developed for sizing mechatronic systems according to energy and dynamic criteria. One of the novelties of this methodology is its structural analysis step. This step enables structural properties to be deduced and helps in the formulation of the specifications. The aim of this paper is to add new graphical procedures to the structural analysis step to determine some structural properties (infinite pole orders and relative orders) from the inverse model (bicausal bond graph model). The structural analysis of the inverse model remains interesting since the essential orders are immediately obtained on the bicausal model. A discussion is carried out regarding the duality between the causal and bicausal procedures.
\end{abstract}

Index Terms-Bond graph, structural analysis, causal path, duality, infinite zero/pole orders, relative order, essential order.

\section{INTRODUCTION}

$\mathbf{T}$ $\mathrm{HE}$ infinite structure analysis of linear time-invariant systems leads to the determination of different invariants such as infinite zero orders, infinite zero order in row (also called relative orders and essential orders), which have been used to solve control problems such as decoupling, disturbance rejection, pole placement, etc. This structure characterization has been established by different approaches: state-space approach [1], [2], [3], [4], [5], geometric approach [6], [7], [8], structured systems [9], [10], [11], [12], graph theory [13], and, more recently, bond graph approach [14], [15], [16], [17]. Moreover, the structural analysis appears interesting in the design and sizing of mechatronic systems. In fact, based on the bond graph language and inverse modelling, a methodology has already been developed for sizing mechatronic systems according to energy and dynamic criteria [18], [19], [20], [21]. One of the original features of this methodology is its structural analysis step: before finding the unknown inputs by simulating the inverse model from the given specified outputs, the I/O causal paths analysis is established to deduce the infinite structure. The different invariants that characterize the infinite structure supply important information on the system structure. For instance, the essential order $n_{i e}$ of the $i^{\text {th }}$ output can be seen as the highest time-differentiation order of the $i^{\text {th }}$ output appearing

The authors are associated with AMPERE laboratory, INSA-Lyon, Villeurbanne, F-69621 FRANCE e-mail: \{firstname $\}.\{$ lastname $\} @$ insalyon.fr,Mariem.El-Feki@ec-lyon.fr in the inverse model of minimal order [2], [22]. Knowing these orders, the outputs $y_{i}$ can be correctly specified: the specifications match with the structure of the given model if and only if, for each output $y_{i}$, the corresponding specification is at least $n_{i e}$ times time-differentiable. The relative order $n_{i}^{\prime}$ can be seen as the minimal number of times it is necessary to time-differentiate the output $y_{i}$ in order to make explicitly appear at least one component of the input's vector in the expression of $y_{i}^{\left(n_{i}^{\prime}\right)}$. Finally, the comparison of the infinite zero orders list with the relative orders list leads to conclude on the decouplability of the model by static-state feedback ${ }^{1}$. The decouplability checking enables designing decouplable systems which are simpler to control instead of designing non-decouplable systems which imply more sophisticated control laws.

With its multidisciplinarity concept and its graphical feature, bond graph language, used in the methodology for sizing mechatronic systems, shows to be an efficient tool for the structural analysis for at least two reasons. Firstly, as bond graph modelling is based on the representation of energy exchanges in the system, the bond graph model intrinsically incorporates the model structure from the energy point of view. Secondly, the state-space and digraph approaches lead to a loss of information about the details of the different physical phenomena involved and the way they are energetically interconnected. On the contrary, the bond graph language makes easy the reading and the physical interpretation of the structural properties.

The aim of this paper is to add to the structural analysis step some new graphical procedures (procedures 6 and 9) to determine some structural properties (infinite pole orders and relative orders) from the inverse model (bicausal bond graph model). These new procedures enable the establishing of the infinite structure analysis on the bicausal bond graph model. The structural analysis of the bicausal model makes easier the determination of the essential orders which plays an important role in the redaction of the specifications. A discussion is also provided on duality between the causal and the bicausal procedures. Bond graph language appears interesting for the duality study. In fact, duality has been used with a certain signification by Birkett and Roe [23] to compare cycle and co-cycle matroids of a bond graph model. Also, Lichiardopol and Sueur [24], [25] established duality,

\footnotetext{
${ }^{1}$ Another method to conclude this is the comparison of the the $i^{t h}$ essential order with the $i^{t h}$ relative order for each output $y_{i}$.
} 
with another signification than the previous one, between controllability and observability of linear systems by the bond graph approach. Here, duality is understood with this second signification.

The proofs of the proposed graphical procedures are based on the exploitation of the determinant of the system matrix of the inverse model. So a bond graph procedure (procedure 2) is proposed to determine graphically this determinant. This procedure corresponds to a translation of the digraph procedure enunciated in [13] and which is adapted to inverse models in this paper (proposition 1 and equation 9). The rules of this translation were established in [26]. Note that a bond graph procedure exists to determine the determinant of the system matrix of the direct model (causal bond graph model), so the proposed procedure is an adaptation of the causal procedure for the inverse model. Moreover, in order to define and prove the bond graph procedure for the determination of infinite pole orders, some intermediary results are proposed:

- theorem 2 for the determination of the infinite pole orders from the system matrix of the inverse model (state-space contribution);

- procedure 4 for the determination of the minors of the system matrix of the inverse model (bond graph contribution);

- procedure 5 for the determination of the highest degree of the minors of order $i$ of the transfer matrix of the inverse model (bond graph contribution).

To introduce and prove the bond graph procedure for the determination of the relative orders from the bicausal model, theorem $\mathbf{5}$ is proposed (state-space contribution). This theorem enables the calculus of the relative orders from the inverse model.

This paper is organized as follows. In Section II, some results on state-space, digraph and bond graph are recalled. The digraph and the bond graph procedures [13], [26] for the determination of the determinant of the system matrix are adapted to the inverse model (proposition 1 and equation 9). In Section III, the causal procedure to find infinite zero orders on the bond graph model is recalled and a new procedure is proposed to find the infinite pole orders of the inverse model on the bicausal bond graph model (procedure 6). Section IV focusses on the causal and the bicausal procedures to determine graphically the relative orders and the essential orders. Indeed, a new procedure to compute relative orders from the inverse model is presented using the state-space approach (theorem 5). Based on this state-space procedure, a bicausal procedure is defined to compute these orders on a bicausal bond graph model (procedure 9). In this paper, the different procedures will be illustrated by a mechanical example and the duality between the causal and bicausal procedures will be discussed. Finally, a conclusion is given in Section V.

\section{FORMALISM PRELIMINARIES}

\section{A. State-space approach}

Let us consider the following square linear time-invariant system $\Sigma$ :

$$
\Sigma:\left\{\begin{array}{l}
\dot{\mathbf{x}}=\mathbf{A x}+\mathbf{B u} \\
\mathbf{y}=\mathbf{C x}+\mathbf{D u}
\end{array}\right.
$$

where:

- $\mathbf{x} \in \mathbb{R}^{n}$ denotes the state vector;

- $\mathbf{u} \in \mathbb{R}^{m}$ (resp. $\mathbf{y} \in \mathbb{R}^{m}$ ) is the input (resp. output) vector.

The associated transfer matrix is supposed strictly proper and defined by:

$$
\mathbf{T}(\mathbf{s})=\mathbf{C}(s \mathbf{I}-\mathbf{A})^{-1} \mathbf{B}+\mathbf{D}
$$

The inverse model is obtained from the direct model $\Sigma$ by successive time-differentiations of each output in order to express the control $\mathbf{u}$ as a function of the state vector and the derivatives of the components of $\mathbf{y}$. So the inverse model can be expressed as:

$$
\left\{\begin{array}{l}
\dot{\mathbf{x}}_{\mathbf{i n v}}(t)=\mathbf{A}_{\mathbf{i n v}} \mathbf{x}_{\mathbf{i n v}}(t)+\mathbf{B}_{\mathbf{i n v}} \mathbf{u}_{\mathbf{i n v}}(t) \\
\mathbf{y}_{\mathbf{i n v}}(t)=\mathbf{C}_{\mathbf{i n v}} \mathbf{x}_{\mathbf{i n v}}(t)+\mathbf{D}_{\mathbf{i n v}} \mathbf{u}_{\mathbf{i n v}}(t)
\end{array}\right.
$$

where

- $\mathbf{x}_{\mathbf{i n v}}(t) \in \mathbb{R}^{n_{\text {inv }}}$ is the state vector;

- $\mathbf{u}_{\text {inv }}(t)=\mathbf{y}_{\alpha}(t) \in \mathbb{R}^{\alpha}\left(\right.$ resp. $\left.\mathbf{y}_{\text {inv }}(t)=\mathbf{u}(t) \in \mathbb{R}^{m}\right)$ is the input (resp. output) vector. The vector $\mathbf{u}_{\text {inv }}(t)=$ $\mathbf{y}_{\alpha}(t)$ is composed of the components of the output vector $\mathbf{y}(\mathbf{t})$ of the direct model $\Sigma$ and their successive derivatives appearing during the construction of the inverse model;

- The matrices $\mathbf{A}_{\mathbf{i n v}}, \mathbf{B}_{\mathbf{i n v}}, \mathbf{C}_{\mathbf{i n v}}$ and $\mathbf{D}_{\text {inv }}$ are constant matrices of respective dimensions $\left(n_{i n v} \times n_{i n v}\right)$, $\left(n_{\text {inv }} \times \alpha\right),\left(m \times n_{\text {inv }}\right)$ and $(m \times \alpha)$.

In the Laplace domain, the inverse model (3) can be written as follows:

$$
\left\{\begin{array}{l}
\mathbf{s} \mathbf{X}_{\mathbf{i n v}}(s)-\mathbf{x}_{\mathbf{i n v}}(0)=\mathbf{A}_{\mathbf{i n v}} \mathbf{X}_{\mathbf{i n v}}(s)+\mathbf{B}_{\mathbf{i n v}}(s) \mathbf{U}_{\mathbf{i n v}}(s) \\
\mathbf{Y}_{\mathbf{i n v}}(s)=\mathbf{C}_{\mathbf{i n v}} \mathbf{X}_{\mathbf{i n v}}(s)+\mathbf{D}_{\mathbf{i n v}}(s) \mathbf{U}_{\mathbf{i n v}}(s)
\end{array}\right.
$$

where $\mathbf{U}_{\text {inv }}(s)=\mathbf{Y}(s) \in \mathbb{R}^{m}$. $\mathbf{B}_{\mathbf{i n v}}(s)$ and $\mathbf{D}_{\text {inv }}(s)$ are polynomial matrices in $s$ of respective dimensions $\left(n_{i n v} \times m\right)$ and $(m \times m)$. $\mathbf{x}_{\mathbf{i n v}}(\mathbf{0})$ is supposed to be zero throughout this paper.

The characteristic polynomial of the matrix $\mathbf{A}_{\text {inv }} \in \mathbb{R}$ is equal to $\operatorname{det}\left(s \mathbf{I}-\mathbf{A}_{\mathbf{i n v}}\right)$. This polynomial can be expressed as follows:

$$
P_{\mathbf{A}_{\mathbf{i n v}}}(s)=s^{n_{i n v}}+\sum_{i=1}^{n_{\text {inv }}} \alpha_{i} s^{n_{i n v}-i}
$$

The transfer matrix $\mathbf{T}_{\mathbf{i n v}}(s)$ of the inverse model is defined by:

$\mathbf{T}_{\mathbf{i n v}}(\mathbf{s})=\mathbf{C}_{\mathbf{i n v}}\left(\mathbf{s I}-\mathbf{A}_{\mathbf{i n v}}\right)^{-1} \mathbf{B}_{\mathbf{i n v}}(s)+\mathbf{D}_{\mathbf{i n v}}(s)$ 
The matrix $\mathbf{T}_{\mathbf{i n v}}(\mathbf{s})$ is a rational matrix but it is not proper. The system matrix $\mathbf{P}_{\text {inv }}(s) \in \mathbb{R}^{\left(n_{i n v}+m\right) \times\left(n_{i n v}+m\right)}$ of the inverse model $\Sigma^{-1}$ is defined by:

$$
\mathbf{P}_{\text {inv }}(s)=\left[\begin{array}{cc}
s \mathbf{I}-\mathbf{A}_{\mathbf{i n v}} & \mathbf{B}_{\mathbf{i n v}}(s) \\
-\mathbf{C}_{\mathbf{i n v}} & \mathbf{D}_{\mathbf{i n v}}(s)
\end{array}\right]
$$

\section{B. Digraph approach}

In this subsection, a digraph model called the 'Laplace digraph' representing the inverse model (4) is defined and a procedure to compute the determinant of the system matrix $\mathbf{P}_{\text {inv }}(s)$ is given. This procedure will be used to define the bond graph procedure to compute the determinant of the system matrix $\mathbf{P}_{\text {inv }}(s)$ of the inverse model (Section II-C). It uses the transition rules [26] from a digraph to a bond graph model.

The Laplace digraph is an adaptation of the classical digraph [27], [13] to the inverse model where the weights of some edges can be functions of the Laplace variable $s$.

Proposition 1. Let us consider the inverse model described by (4), the associated digraph is characterized by:

- $a$ vertex set $V=U_{i n v} \cup X \cup Y_{\text {inv }}$ where $U_{i n v}=$ $\left\{y_{1}, \ldots, y_{m}\right\}, \quad X=\left\{x_{1}, \ldots, x_{n_{i n v}}\right\}$ and $Y_{i n v}=$ $\left\{u_{1}, \ldots, u_{m}\right\}$ are respectively the sets of input, state and output vertices;

- an edge set $E=E_{\mathbf{A}_{\text {inv }}} \cup E_{\mathbf{B}_{\text {inv }}(s)} \cup E_{\mathbf{C}_{\text {inv }}} \cup E_{\mathbf{D}_{\text {inv }}(s)}$ with:

$E_{\mathbf{A}_{\text {inv }}}=\left\{\left(x_{j}, x_{i}\right) \mid a_{i j} \neq 0\right\}$

$E_{\mathbf{B}_{\text {inv }}(s)}=\left\{\left(y_{j}, x_{i}\right) \mid b_{i j}(s) \neq 0\right\}$

$E_{\mathbf{C}_{\text {inv }}}=\left\{\left(x_{j}, u_{i}\right) \mid c_{i j} \neq 0\right\}$

$E_{\mathbf{D}_{\text {inv }}(s)}=\left\{\left(y_{j}, u_{i}\right) \mid d_{i j}(s) \neq 0\right\}$

where $\left(x_{j}, x_{i}\right)$ denotes a directed edge from the vertex $x_{j} \in X$ to the vertex $x_{i} \in X$ and $a_{i j} \neq 0$ means that the $(i, j)^{\text {th }}$ entry of the matrix $\mathbf{A}_{\mathbf{i n v}}$ is not fixed to zero (of course, the same reasoning is applied to the sets $E_{\mathbf{B}_{\text {inv }}(s)}, E_{\mathbf{C}_{\mathbf{i n v}}}$ and $\left.E_{\mathbf{D}_{\text {inv }}(s)}\right)$. Each edge $\left(x_{j}, x_{i}\right)$ (resp. $\left.\left(y_{j}, x_{i}\right),\left(x_{j}, u_{i}\right),\left(y_{j}, u_{i}\right)\right)$ has a weight equal to the $(i, j)^{t h}$ entry of the matrix $\mathbf{A}_{\mathbf{i n v}}$ (resp. $\mathbf{B}_{\mathbf{i n v}}(s), \mathbf{C}_{\mathbf{i n v}}$, $\left.\mathbf{D}_{\text {inv }}(s)\right)$. Consequently, the weights of the edges $\left(y_{j}, x_{i}\right)$ and $\left(y_{j}, u_{i}\right)$ are functions of the Laplace variable $s$.

The exploitation rules of the Laplace digraph representing the inverse model (4) are the same as those of the classical digraph.

Now, let us recall some definitions which are necessary for the determination, from a digraph, of the determinant of the system matrix $\mathbf{P}(s)$ of the system $\Sigma$.

Definition 1. A (directed) path is a sequence of edges such that the initial vertex of the succeeding edge is the final vertex of the preceding edge [27], [13].

Definition 2. The weight of a path is equal to the product of the weights of the edges composing the sequence [27].

Definition 3. A feedback edge is an edge of weight (-1) between an output vertex and an input vertex [13].
Definition 4. A digraph cycle is a path whose initial and final vertices are the same [13].

Definition 5. The weight of a digraph cycle is equal to the weight of the corresponding closed path [13].

Definition 6. Two cycles are said to be disjoint if there is neither a vertex nor an edge in common [13].

Definition 7. A cycle family is a set of disjoint cycles [13].

Definition 8. The weight of a cycle family is equal to the product of the weights of the digraph cycles composing that family [13].

Procedure 1. Let us consider $\mathcal{G}(\Sigma)$ the digraph associated to the system $\Sigma$. The determinant of the system matrix $\mathbf{P}(s)$ is then given by the following expression [13]:

$$
\operatorname{det}(\mathbf{P}(s))=\sum_{k=0}^{n} \rho_{k}^{\{m\}} s^{n-k}
$$

where:

- $n$ corresponds to the number of state vertices and $m$ corresponds to the number of input (resp. output) vertices;

- $\mathcal{G}\left(\Sigma^{\prime}\right)$ is the digraph obtained from the digraph $\mathcal{G}(\Sigma)$ by adding feedback edge between each output vertex and each input vertex;

- $\rho_{k}^{\{m\}}$ corresponds, on the digraph $\mathcal{G}\left(\Sigma^{\prime}\right)$, to the sum of the weights of each cycle family containing $k$ state vertices and at least $m$ feedback edges. In this calculation, the weight of each cycle family must be multiplied by $(-1)^{d}$ where $d$ is the number of disjoint cycles contained in the cycle family such $d \geq m$. The weight of each family must be multiplied also by $(-1)^{\sigma_{k}}$ where $\sigma_{k}$ is the number of permutations needed to order the output vertices of the cycle family in the initial order of the output vertices when the cycles of the family are ordered in the initial order of the input vertices.

Eq. (9) can be adapted to the inverse model described by (4):

$$
\operatorname{det}\left(\mathbf{P}_{\mathbf{i n v}}(s)\right)=\sum_{k=0}^{n_{\text {inv }}} \rho_{k}^{\{m\}}(s) s^{n_{\text {inv }}-k}
$$

where:

- the number of state vertices is equal to $n_{i n v}$;

- the sum of the weights of each cycle family containing $k$ state vertices and at least $m$ feedback edges depends on the Laplace variable $s$.

This direct deduction is possible because the only difference between the digraph representing the inverse model and the digraph representing the direct model is the appearance of the Laplace variable in the weights of some edges $\left(\left(y_{j}, x_{i}\right)\right.$ and $\left.\left(y_{j}, u_{i}\right)\right)$ on the Laplace digraph. Otherwise this changes nothing in the weight calculation (see [13]) and consequently in the calculation of the determinant of the system matrix $\mathbf{P}_{\text {inv }}(s)$. 
Example. Let us consider the inverse model described by the following system:

$$
\left\{\begin{array}{l}
\dot{\mathbf{q}}=\mathbf{y}_{\mathbf{1}}-\mathbf{y}_{\mathbf{2}} \\
\mathbf{u}_{\mathbf{1}}=\frac{1}{C} \mathbf{q}+I_{1} \dot{\mathbf{y}}_{\mathbf{1}}+R \mathbf{y}_{\mathbf{1}}-R \mathbf{y}_{\mathbf{2}} \\
\mathbf{u}_{\mathbf{2}}=-\frac{1}{C} \mathbf{q}+I_{2} \dot{\mathbf{y}}_{\mathbf{2}}-R \mathbf{y}_{\mathbf{1}}+R \mathbf{y}_{\mathbf{2}}
\end{array}\right.
$$

In the Laplace domain, this model is characterized by the following matrices: $\mathbf{A}_{\mathbf{i n v}}=0, \quad \mathbf{B}_{\mathbf{i n v}}(s)=\left[\begin{array}{ll}1 & -1\end{array}\right]$, $\mathbf{C}_{\mathbf{i n v}}=\left[\begin{array}{c}\frac{1}{C} \\ -\frac{1}{C}\end{array}\right]$ and $\mathbf{D}_{\mathbf{i n v}}(s)=\left[\begin{array}{cc}I_{1} s+R & -R \\ -R & I_{2} s+R\end{array}\right]$.

The associated digraph is given in Fig. 1.

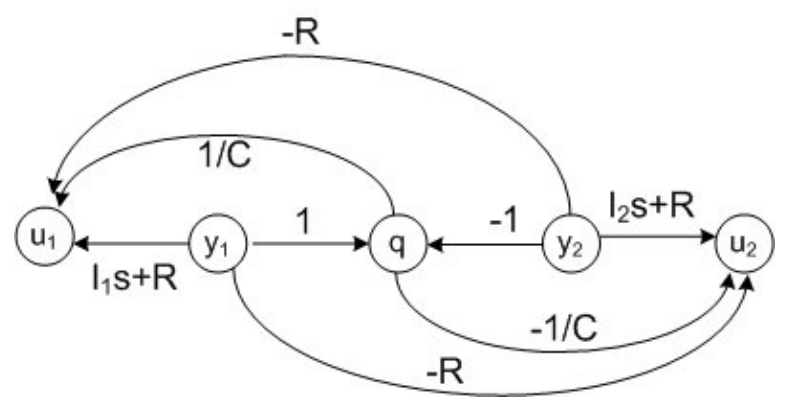

Fig. 1. Example of the digraph representation of an inverse model.

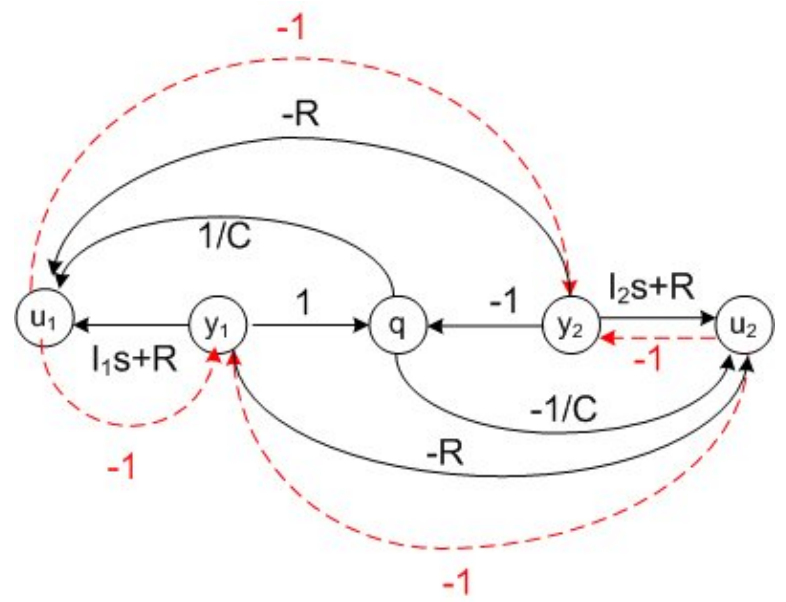

Fig. 2. The digraph $\mathcal{G}\left(\Sigma^{\prime}\right)$ for the determination of the determinant of the system matrix $\mathbf{P}_{\text {inv }}(s)$.

The determinant of the system matrix of the inverse model (10) found by the state-space approach is expressed as follows:

$$
\operatorname{det}\left(\mathbf{P}_{\mathbf{i n v}}(s)\right)=I_{1} I_{2} s^{3}+R\left(I_{1}+I_{2}\right) s^{2}+\frac{\left(I_{1}+I_{2}\right)}{C} s
$$

Now, let us compute this determinant by applying the digraph procedure (9). The digraph $\mathcal{G}\left(\Sigma^{\prime}\right)$ (digraph with feedback edges) is given in Fig. 2. The analysis of the cycle families is detailed in Fig. 3 and can be summarized in the following table:

\begin{tabular}{|c|c|c|c|}
\hline Family & $\rho_{k}^{\{m\}}(s) \cdot s^{n_{i n v}-k}$ & $d$ & $\sigma_{k}$ \\
\hline $\mathcal{F}_{1}$ & $\left(-\left(I_{1} s+R\right)\right) \cdot\left(-\left(I_{2} s+R\right)\right) \cdot s^{(1-0)}$ & 2 & 0 \\
\hline $\mathcal{F}_{2}$ & $\left(-\left(I_{1} s+R\right)\right) \cdot\left(-\frac{1}{C}\right) \cdot s^{(1-1)}$ & 2 & 0 \\
\hline $\mathcal{F}_{3}$ & $\left(\frac{1}{C}\right) \cdot\left(I_{2} s+R\right) \cdot s^{(1-1)}$ & 2 & 0 \\
\hline $\mathcal{F}_{4}$ & $\left(\frac{1}{C}\right) \cdot(R) \cdot s^{(1-1)}$ & 2 & 1 \\
\hline $\mathcal{F}_{5}$ & $(R) \cdot(R) \cdot s^{(1-0)}$ & 2 & 1 \\
\hline $\mathcal{F}_{6}$ & $(R) \cdot\left(\frac{1}{C}\right) \cdot s^{(1-1)}$ & 2 & 1 \\
\hline
\end{tabular}

The expression of the determinant of the system matrix $\mathbf{P}_{\text {inv }}(s)$ (11) is, hence, determined graphically.

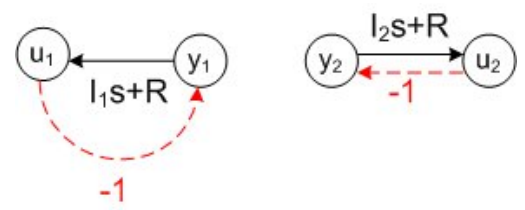

(a) Family $\mathcal{F}_{1}$

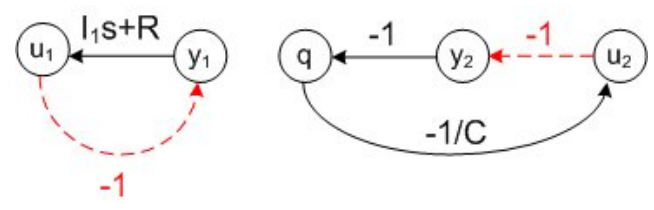

(b) Family $\mathcal{F}_{2}$

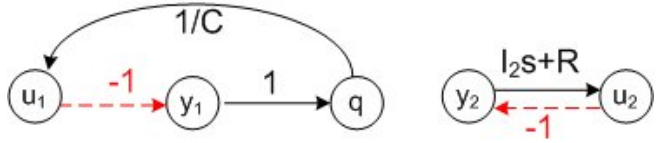

(c) Family $\mathcal{F}_{3}$

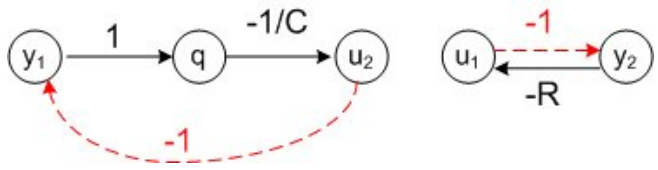

(d) Family $\mathcal{F}_{4}$

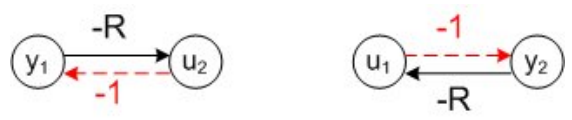

(e) Family $\mathcal{F}_{5}$

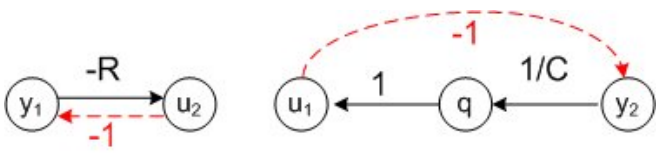

(f) Family $\mathcal{F}_{6}$

Fig. 3. Analysis of cycle families.

\section{Bond graph approach}

According to the bond graph approach, the inverse model is obtained from the bicausal bond graph model. The invertibility conditions were discussed in [21], [28] and the inversion procedure was presented in [29]. Let us consider a bond graph model representing the system $\Sigma$ as in (1) and a bicausal bond graph model representing its inverse model as in (3) and in (4).

Definition 9. In a bond graph model, a causal path is a series of effort and flow variables successively related according to the model causality assignment [15], [21]. 
Definition 10. In a causal bond graph model, an input/output (I/O) causal path starts from a modulated element (input) and goes to a detector (output) [15], [21].

Definition 11. In a bicausal bond graph model, an Input/Output (I/O) causal path is a causal path starts from a double source $S_{e} S_{f}$ (input) and goes to a double detector $D_{e} D_{f}$ (output).

Definition 12. In a bond graph model, two causal paths are said to be different only if there is no storage element (I or C) in integral causality in common [14].

Definition 13. In a bond graph model, two causal paths are said to be disjoint only if there is no variable in common [21]. This translates, by a graphical disjunction of these two causal paths, into the bond graph model.

Definition 14. In a bond graph model, the order $\omega_{k}\left(v_{i} \rightarrow v_{j}\right)$ (or the generalized length) of a causal path $\mathbb{k}$ between a variable $v_{i}$ and another variable $v_{j}$ is equal to the difference between the number of energy storages in integral causality and the number of energy storages in derivative causality met along this path [19] [17].

Definition 15. The gain $G(s)$ (resp. static gain $G$ ) of a causal path is defined by [30]:

$$
G(s)=(-1)^{n_{0}+n_{1}} \prod_{i}\left(m_{i}\right)^{k_{i}} \prod_{j}\left(r_{j}\right)^{l_{j}} \prod_{e} g_{e}(s)
$$

where:

- $n_{0}$ corresponds to the total number of the orientation switches in 0 junctions when the flow variable is followed;

- $n_{1}$ corresponds to the total number of the orientation switches in 1 junctions when the effort variable is followed;

- $m_{i}$ corresponds to the gain of the elements $T F_{i}$ along the causal path, with $k_{i}$ equal to $(+1)$ or $(-1)$ according to the causality on the transformer;

- $r_{j}$ corresponds to the gain of the elements $G Y_{j}$ along the causal path, with $l_{i}$ equal to $(+1)$ or $(-1)$ according to the causality on the gyrator;

- $\prod_{e} g_{e}(s)$ corresponds to the product of the gains (resp. static gains $\left.g_{e}\right)$ of the elements $R, I$ and $C$ of the causal path.

Definition 16. A causal cycle is a closed causal path which can contain several distinct storage elements [26].

Definition 17. The gain (resp. static gain) of a causal cycle is equal to the gain (resp. static gain) of the corresponding closed causal path [26].

Definition 18. A bond graph family is a set of different causal cycles and I/O causal paths [31].

Definition 19. The gain (resp. static gain) of a bond graph family is equal to the product of the gains (resp. static gains) of the causal cycles and causal paths composing the considered family [31].

Definition 20. The order of a bond graph family is equal to the difference between the number of energy storages in integral causality and the number of energy storages in derivative causality composing this family.

In this paper, the dynamic elements (I and $\mathrm{C}$ ) of the bond graph models (causal and bicausal bond graph models) are supposed independent. From these bond graph definitions and from (9), a bond graph procedure is proposed and shown to determine the determinant of the system matrix $\mathbf{P}_{\mathbf{i n v}}(s)$.

Procedure 2. In a bicausal bond graph model, the determinant of the system matrix $\mathbf{P}_{\mathbf{i n v}}(s)$ is given by the following expression:

$$
\operatorname{det}\left(\mathbf{P}_{\mathbf{i n v}}(s)\right)=\sum_{k=-n_{D}}^{n_{\text {inv }}} \rho_{k}^{\{m\}} s^{n_{i n v}-k}
$$

where in the bicausal bond graph model:

- $n_{\text {inv }}\left(\right.$ resp. $\left.n_{D}\right)$ corresponds to the number of storage elements in integral causality (resp. in derivative causality) and $m$ corresponds to the number of inputs/outputs ;

- $\rho_{k}^{\{m\}}$ corresponds to the sum of the static gains of bond graph families of order $k$ and containing $m$ different $I / O$ causal paths. In this calculation, the static gain of each bond graph family must be multiplied by $(-1)^{d}$ where $d$ is the number of cycles contained in the bond graph family such $d \geq m$. Also, the static gain of each family must be multiplied by $(-1)^{\sigma_{k}}$ where $\sigma_{k}$ is the number of necessary permutations to order the outputs in the initial order of the output vector when the $m$ I/O causal paths of the family are ordered in the initial order of the input vector.

Proof. Let us consider (9) defined for the determination of the determinant of the system matrix $\mathbf{P}_{\text {inv }}(s)$ from a digraph $\mathcal{G}\left(\Sigma^{-1}\right)$. By exploiting the existing correspondence between the disjoint cycles containing feedback edges on the digraph and the different I/O causal paths on the bond graph model [26], the following result can be deduced:

$$
\operatorname{det}\left(\mathbf{P}_{\mathbf{i n v}}(s)\right)=\sum_{l=0}^{n_{\text {inv }}} \rho_{l}^{\{m\}}(s) s^{n_{i n v}-l}
$$

where in the bicausal bond graph model, $\rho_{l}^{\{m\}}(s)$ corresponds to the sum of the gains of families containing $l$ energy storages in integral causality and $m$ different $\mathrm{I} / \mathrm{O}$ causal paths. The gain of each family is computed as follows: if the traversed storage element is in integral causality then the static gain is considered and if the traversed storage element is in derivative causality then the gain is considered. So, the appearance of the Laplace variable in the gain of the family is due to the storage elements in derivative causality met along the bond graph family. Due to this, it is more interesting to consider the order $k$ of the bond graph family and its static gains $\rho_{k}^{\{m\}}$. Moreover, the lowest order of each family is $-n_{D}$ (the total number of storage elements in derivative causality) and the highest, $n_{i n v}$ (the total number of storage elements in integral causality). Thus (14) is equivalent to (13). End of proof. 
Example. Let us consider the bicausal bond graph illustrated in Fig. 4 and corresponding to the system of equations (10). The analysis of the bond graph families is detailed in Fig. 5 and Fig. 6 and can be summarized by the following table:

\begin{tabular}{|c|c|c|c|c|}
\hline Family & $\mathrm{k}$ & $\rho_{k}^{\{m\}}(s) \cdot s^{n_{\text {inv }}-k}$ & $d$ & $\sigma_{k}$ \\
\hline $\mathcal{F}_{1}$ & -2 & $I_{1} I_{2} \cdot s^{1-(-2)}$ & 0 & 0 \\
\hline $\mathcal{F}_{2}$ & -1 & $R I_{2} \cdot s^{1-(-1)}$ & 0 & 0 \\
\hline $\mathcal{F}_{3}$ & -1 & $R I_{1} \cdot s^{1-(-1)}$ & 0 & 0 \\
\hline $\mathcal{F}_{4}$ & 0 & $\frac{I_{1}}{C} \cdot s^{1-0}$ & 0 & 0 \\
\hline $\mathcal{F}_{5}$ & 0 & $R^{2} \cdot s^{1-0}$ & 0 & 0 \\
\hline $\mathcal{F}_{6}$ & 0 & $R^{2} \cdot s^{1-0}$ & 0 & 1 \\
\hline $\mathcal{F}_{7}$ & 0 & $\frac{I_{2}}{C} \cdot s^{1-0}$ & 0 & 0 \\
\hline $\mathcal{F}_{8}$ & 1 & $\frac{R}{C} \cdot s^{1-1}$ & 0 & 0 \\
\hline $\mathcal{F}_{9}$ & 1 & $\frac{R}{C} \cdot s^{1-1}$ & 0 & 0 \\
\hline $\mathcal{F}_{10}$ & 1 & $\frac{R}{C} \cdot s^{1-0}$ & 0 & 1 \\
\hline $\mathcal{F}_{11}$ & 1 & $\frac{R}{C} \cdot s^{1-0}$ & 0 & 1 \\
\hline
\end{tabular}

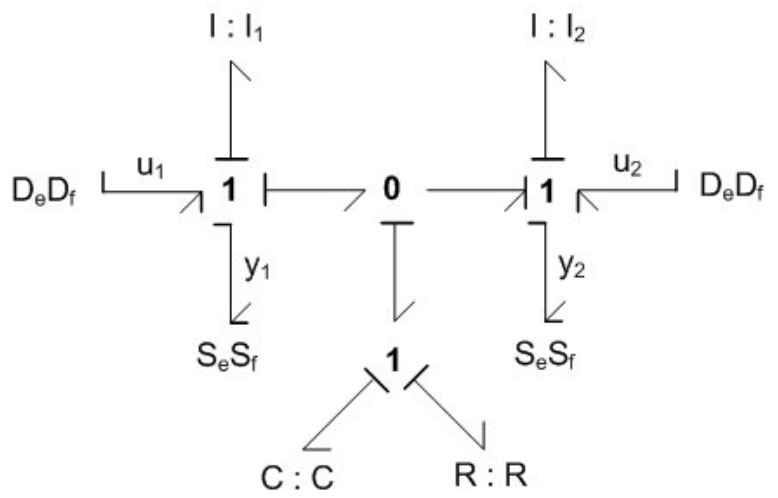

Fig. 4. Example of bicausal bond graph model.

So the determinant of the system matrix $\mathbf{P}_{\mathbf{i n v}}(s)$ (11) is determined from the bicausal bond graph model.

\section{INFINITE ZERO/POLE ORDERS}

\section{A. State-space approach}

In this subsection, some state-space results on infinite zero/pole orders are recalled. After that, the relationship between the infinite pole orders of the transfer matrix $\mathbf{T}_{\mathbf{i n v}}(s)$ (resp. of a sub-matrix $\mathbf{T}_{\mathbf{i n v}}(s)_{h_{1}, \ldots, h_{i}}^{j_{1}, \ldots, j_{i}}$ of the transfer matrix) and the order of the system matrix $\mathbf{P}_{\text {inv }}(s)$ (resp. of a sub-matrix $\mathbf{P}_{\text {inv }}(s)_{1, \ldots, n_{i n v}, n_{i n v}+h_{1}, \ldots, n_{i n v}+h_{i}}^{1, \ldots, n_{i n v}, n_{i n v}+j_{1}, \ldots, n_{i n v}+j_{i}}$ of the system matrix) will be given.

Definition 21. The degree of a rational function $F(s)=\frac{N(s)}{D(s)}$ is defined by [32]:

$$
\operatorname{deg}(F(s))=\operatorname{deg}(N(s))-\operatorname{deg}(D(s))
$$

Theorem 1. For any $(p \times m)$ rational matrix $G(s)$ (not strictly proper or even proper) of rank $r$, the Smith-MacMillan factorization at infinity is defined by [4], [32]:

$$
G(s)=B_{1}(s)\left[\begin{array}{cc}
\Delta(s) & 0 \\
0 & 0
\end{array}\right] B_{2}(s)
$$

with $B_{1}(s)$ and $B_{2}(s)$ biproper matrices (proper and with proper inverse): $\operatorname{det}\left(\lim _{s \rightarrow \infty} B_{i}(s)\right) \neq 0, i=\{1,2\}$ and $\Delta(s)=$ $\operatorname{diag}\left(s^{t_{1}}, \ldots, s^{t_{r}}\right), t_{1} \geq \ldots \geq t_{r} . t_{i} ; i=\{1, \ldots, r\} ;$ can be computed as a function of the minors of $G(s)$ :

$$
t_{i}=\delta_{i}-\delta_{i-1}
$$

where $\delta_{i}$ is the highest degree of the minors of order $i$ of $G(s)$, $\delta_{0}=0$.

- If $t_{i}>0$ then $n_{i}=t_{i}$ is an order of a pole at infinity;

- If $t_{i}<0$ then $n_{i}=-t_{i}$ is an order of a zero at infinity.

The transfer matrix $\mathbf{T}(\mathbf{s})$ of the system $\Sigma$ has only zeros at infinity [1]. So, the transfer matrix $\mathbf{T}_{\mathbf{i n v}}(s)$ of the inverse model (3) has only poles at infinity ${ }^{2}$. So, let us propose a state-space procedure to compute the orders of these poles at infinity from the system matrix $\mathbf{P}_{\text {inv }}(s)$.

Theorem 2. Let us consider $\mathbf{T}_{\mathbf{i n v}}(s)_{h_{1}, \ldots, h_{i}}^{j_{1}, \ldots, j_{i}} \in \mathbb{R}^{i \times i}$ the sub-matrix obtained from the matrix $\mathbf{T}_{\mathbf{i n v}}(s)$ by selecting the rows $h_{1}, \ldots, h_{i}$ and the columns $j_{1}, \ldots, j_{i}$ such that $\operatorname{det}\left(\mathbf{T}_{\mathbf{i n v}}(s)_{h_{1}, \ldots, h_{i}}^{j_{1}, \ldots, j_{i}}\right) \neq 0$ and $\delta_{i_{h_{1}, \ldots, h_{i}}, \ldots, j_{i}}$ is the degree of this determinant ${ }^{3}$, then:

$$
\delta_{i_{h_{1}, \ldots, h_{i}}^{j_{1}, \ldots, j_{i}}}=\beta_{i_{h_{1}, \ldots, h_{i}}^{j_{1}, \ldots, j_{i}}}-n_{i n v}
$$

where:

- $n_{\text {inv }}$ is the state dimension of the inverse model ;

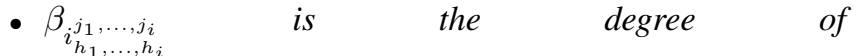
$\operatorname{det}\left(\mathbf{P}_{\text {inv }}(s)_{1, \ldots, n_{\text {inv }}, n_{i n v}+j_{1}, \ldots, n_{\text {inv }}+j_{i}}^{1, \ldots, n_{\text {inv }}+h_{1}, \ldots, n_{\text {inv }}+h_{i}}\right)$;

- $\mathbf{P}_{\text {inv }}(s)_{1, \ldots, n_{i n v}, n_{i n v}+h_{1}, \ldots, n_{i n v}+h_{i}}^{1, \ldots, n_{i n v}, n_{i n v}} \in \mathbb{R}^{\left(n_{i n v}+i\right) \times\left(n_{i n v}+i\right)}$ is deduced from the system matrix $\mathbf{P}_{\mathbf{i n v}}(s)$ by selecting only the rows $1, \ldots, n_{\text {inv }}, n_{\text {inv }}+h_{1}, \ldots, n_{\text {inv }}+h_{i}$ and the columns $1, \ldots, n_{i n v}, n_{i n v}+j_{1}, \ldots, n_{i n v}+j_{i}$ (the $n_{\text {inv }}$ first rows of the $n_{\text {inv }}$ first columns of $\mathbf{P}_{\mathbf{i n v}}(s)$ are conserved).

Furthermore, $\delta_{i_{h_{1}, \ldots, h_{i}}^{j_{1}, \ldots, j_{i}}}$ is necessarily maximal for $\beta_{i_{h_{1}, \ldots, h_{i}}^{j_{1}, \ldots, j_{i}}}$ maximal, thus

$$
\delta_{i}=\beta_{i}-n_{\text {inv }}
$$

where $\delta_{i}=\max _{j_{1}, \ldots, j_{i} \in(\llbracket 1, m \rrbracket)^{i}}\left\{\delta_{i_{h_{1}}^{j_{1}, \ldots, h_{i}}}\right\}$ the highest de$h_{1}, \ldots, h_{i} \in(\llbracket 1, m \rrbracket)^{i}$

gree of the minors of order $i$ of $\mathbf{T}_{\mathbf{i n v}}(s)$ and $\beta_{i}=$ $\max _{j_{i} \in(\llbracket 1, m \rrbracket)^{i}}\left\{\beta_{i_{h_{1}}^{j_{1}, \ldots, h_{i}}, \ldots, j_{i}}\right\}$ is the highest degree of the $j_{1}, \ldots, j_{i} \in(\llbracket 1, m \rrbracket)^{i}$

minors of order $i$ of $\mathbf{P}_{\mathbf{i n v}}(s)$.

Proof. By applying Schur's formula, the determinant of the system matrix can be expressed as:

$$
\operatorname{det}\left(\mathbf{P}_{\mathbf{i n v}}(s)\right)=P_{\mathbf{A}_{\mathbf{i n v}}}(s) \operatorname{det}\left(\mathbf{T}_{\mathbf{i n v}}(s)\right)
$$

So, by generalizing to the minors, the following result is obtained:

$$
\begin{aligned}
& \operatorname{det}\left(\mathbf{P}_{\mathbf{i n v}}(s)_{1, \ldots, n_{i n v}, n_{i n v}+h_{1}, \ldots, n_{i n v}+h_{i}}^{1, \ldots, n_{i n v}+n_{1}}\right) \\
& =P_{\mathbf{A}_{\text {inv }}}(s) \operatorname{det}\left(\mathbf{T}_{\mathbf{i n v}}(s)_{h_{1}, \ldots, h_{i}}^{j_{1}, \ldots, j_{i}}\right)
\end{aligned}
$$

\footnotetext{
${ }^{2}$ This result can be deduced directly from the Smith-MacMillan factorization at infinity of the transfer matrix of the direct model $\Sigma$.

${ }^{3}$ The zero minors of $\mathbf{T}_{\mathbf{i n v}}(s)$ have no effect on the infinity structure.
} 


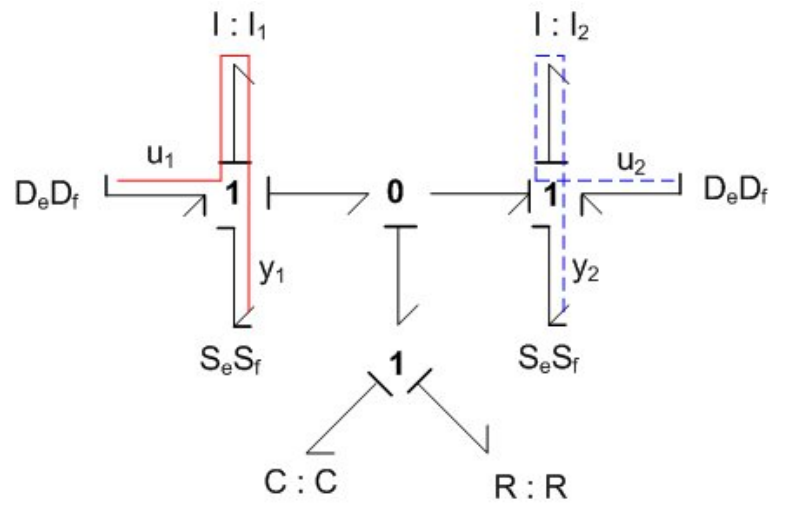

(a) Family $\mathcal{F}_{1}$

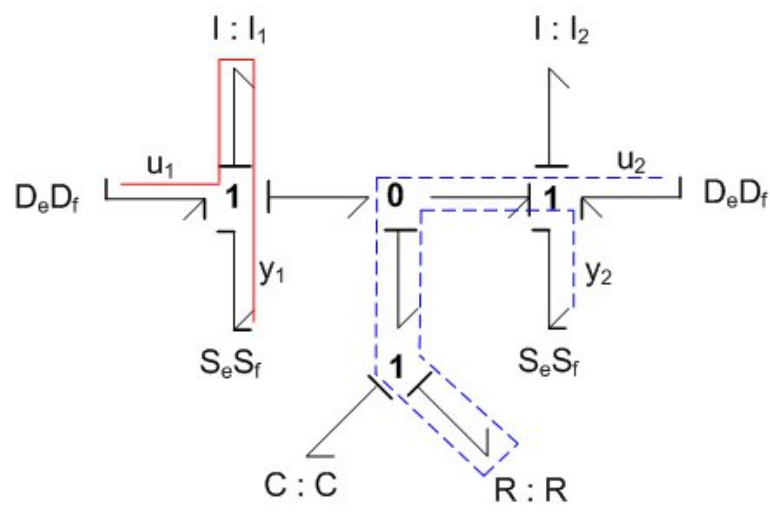

(c) Family $\mathcal{F}_{3}$

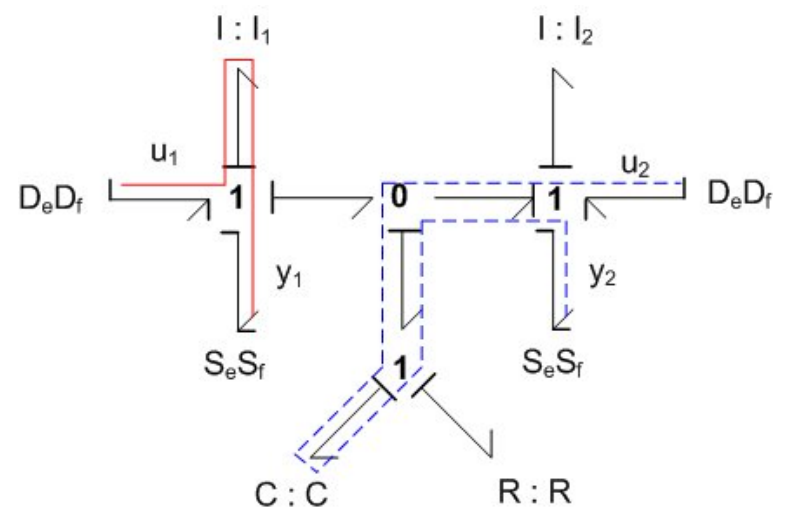

(e) Family $\mathcal{F}_{5}$

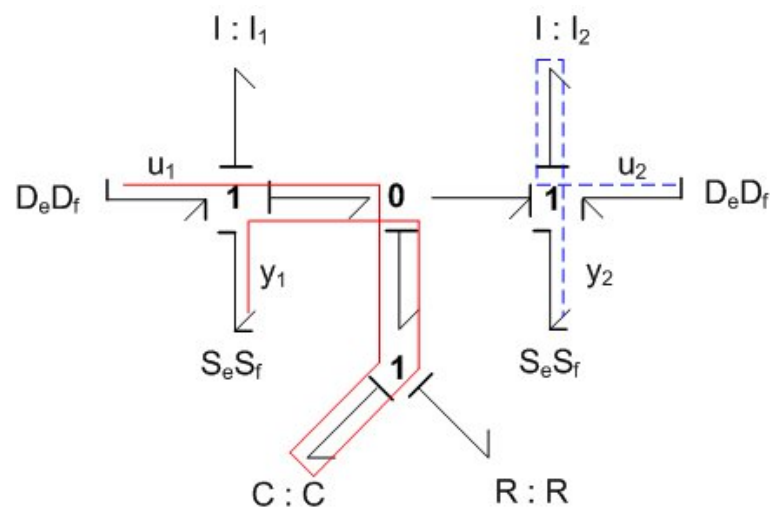

(g) Family $\mathcal{F}_{7}$

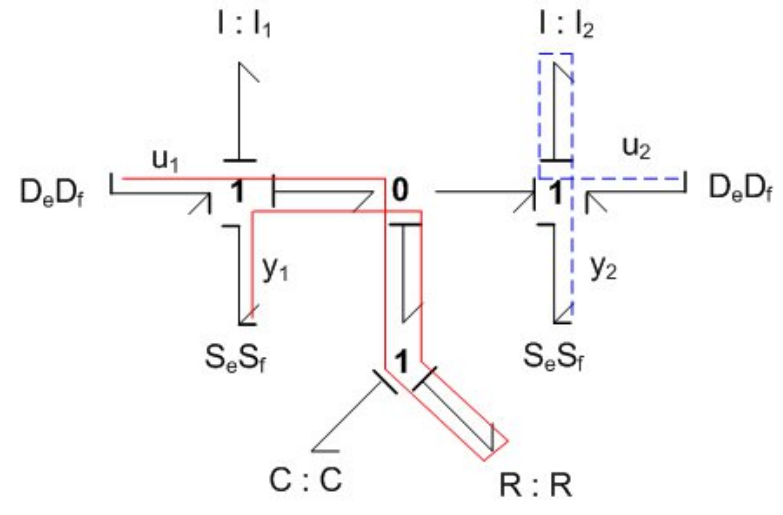

(b) Family $\mathcal{F}_{2}$

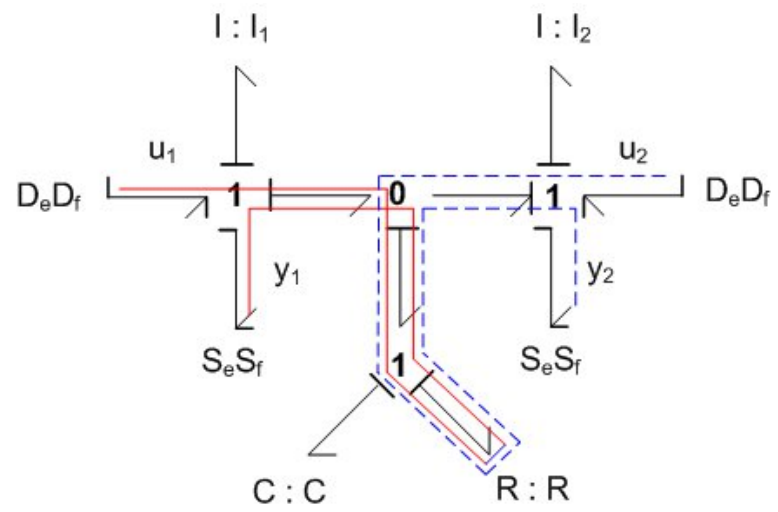

(d) Family $\mathcal{F}_{4}$

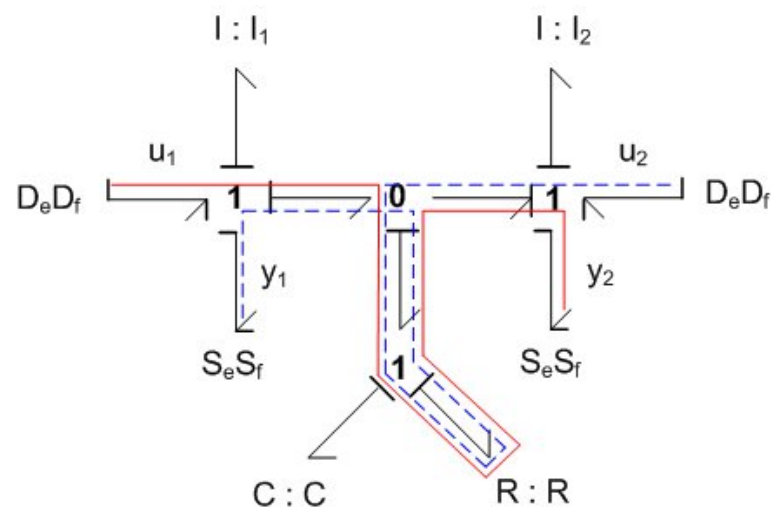

(f) Family $\mathcal{F}_{6}$

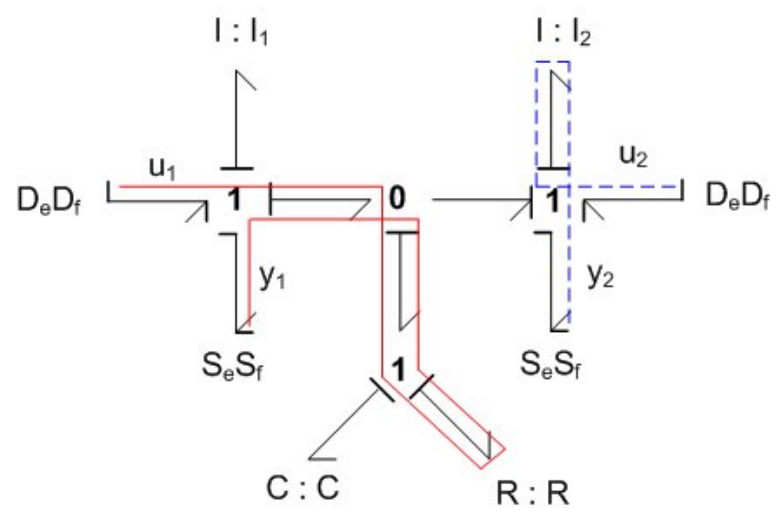

(h) Family $\mathcal{F}_{8}$

Fig. 5. Analysis of bond graph families: $\mathcal{F}_{1} \ldots \mathcal{F}_{8}$. 


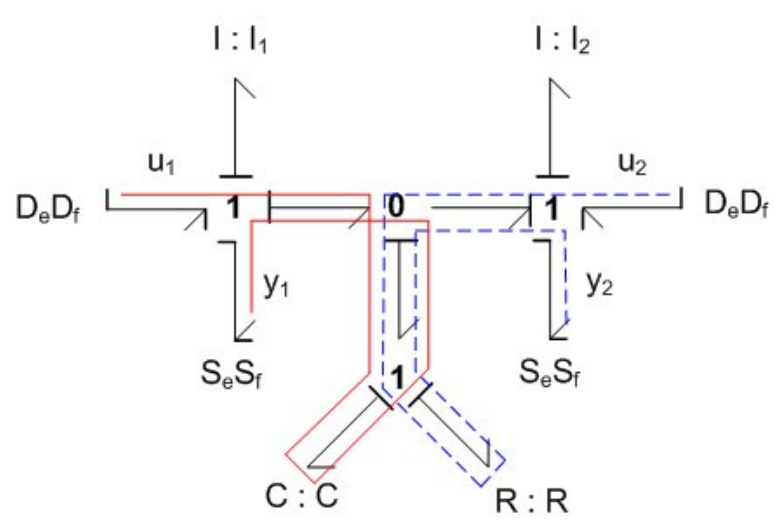

(a) Family $\mathcal{F}_{9}$

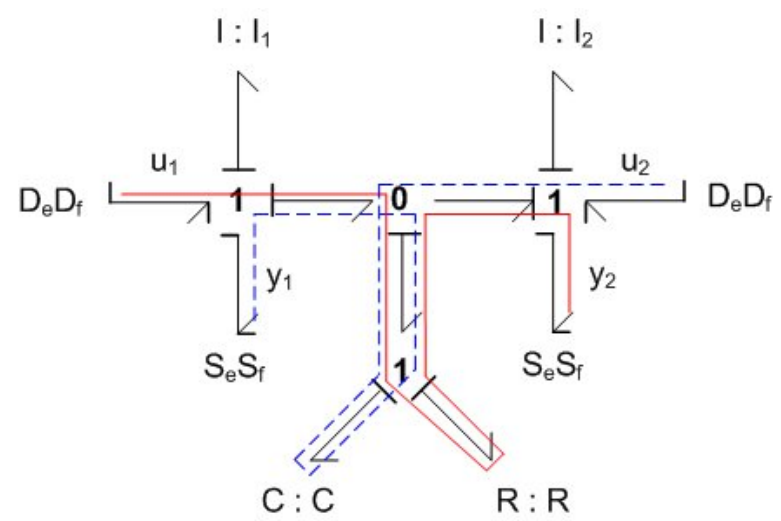

(b) Family $\mathcal{F}_{10}$

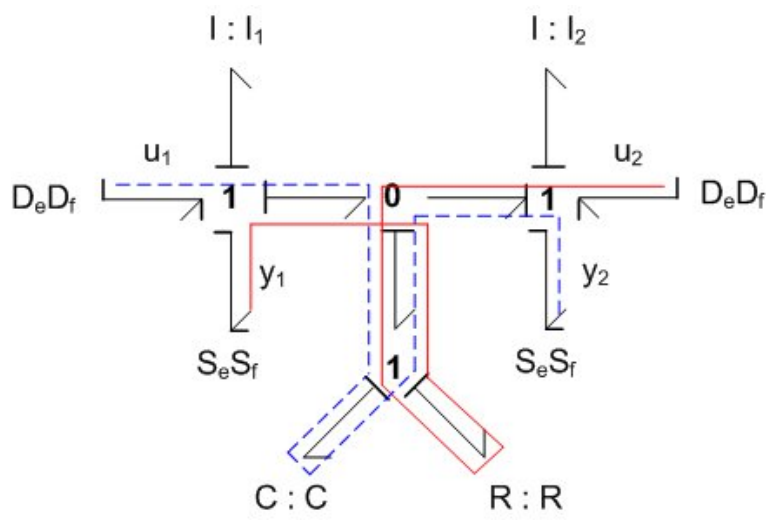

(c) Family $\mathcal{F}_{11}$

Fig. 6. Analysis of bond graph families: $\mathcal{F}_{9} \ldots \mathcal{F}_{11}$.

As $\operatorname{det}\left(\mathbf{T}_{\mathbf{i n v}}(s)_{h_{1}, \ldots, h_{i}}^{j_{1}, \ldots, j_{i}}\right) \neq 0$, then $\operatorname{det}\left(\mathbf{T}_{\mathbf{i n v}}(s)_{h_{1}, \ldots, h_{i}}^{j_{1}, \ldots, j_{i}}\right)$ $s \underset{\sim}{\sim} s^{-n_{i n v}} \operatorname{det}\left(\mathbf{P}_{\text {inv }}(s)_{1, \ldots, n_{i n v}, n_{i n v}+h_{1}, \ldots, n_{i n v}+h_{i}}^{1, \ldots, n_{i n v}, n_{i n v}+j_{1}, \ldots, n_{i n v}+j_{i}}\right)$ because $^{4}$, from (5), $P_{\mathbf{A}_{\text {inv }}}(s) \underset{s \longrightarrow+\infty}{\sim} s^{n_{i n v}}$.

$\mathbf{P}_{\text {inv }}(s)$ is a polynomial matrix, and so $\operatorname{det}\left(\mathbf{P}_{\mathbf{i n v}}(s)_{1, \ldots, n_{i n v}, n_{i n v}+h_{1}, \ldots, n_{i n v}+h_{i}}^{1, \ldots, n_{i n v}, n_{i n v}+j_{1}, \ldots, n_{i n v}+j_{i}}\right)$ is a polynomial in $s$, and, consequently, $\operatorname{det}\left(\mathbf{T}_{\text {inv }}(s)_{h_{1}, \ldots, h_{i}}^{j_{1}, \ldots, j_{i}}\right) \underset{s \longrightarrow+\infty}{\stackrel{\sim}{\longrightarrow}}$ $s^{-n_{i n v}+\operatorname{deg}\left(\operatorname{det}\left(\mathbf{P}_{\mathbf{i n v}}(s)_{1, \ldots, n_{i n v}, n_{i n v}+h_{1}, \ldots, n_{i n v}+h_{i}}^{1, \ldots, n_{i n v}, n_{i n v}+j_{1}, \ldots, n_{i n v}+j_{i}}\right)\right)}$. Thus, it can be deduced: $\delta_{i_{h_{1}, \ldots, h_{i}}^{j_{1}, \ldots, j_{i}}}=\beta_{i_{h_{1}, \ldots, h_{i}}^{j_{1}, \ldots, j_{i}}}-n_{i n v}$ and for the $\operatorname{maximas} \delta_{i}=\beta_{i}-n_{\text {inv }}$.

End of proof.

Note. Theorem 2 will be used in the proof of the bicausal procedure 9 for the determination of the infinite pole orders (Section III-C).

\section{B. Bond graph approach: Causal procedure}

The causal procedure is applied to the bond graph model in preferential integral causality to compute the infinite zero orders of the direct model $\Sigma$.

Procedure 3. Let us consider a bond graph model representing a linear time-invariant system as in (1) with $\mathbf{D}=\mathbf{0}$. The system $\Sigma$ is supposed square and invertible so the number of

\footnotetext{
${ }^{4}$ the notation $\underset{s \longrightarrow+\infty}{\sim}$ represents the limit as $s$ tends to $+\infty$.
}

the infinite zero orders is equal to $m$. Moreover, each infinite zero order $n_{i}$ can be computed as follows [33]:

$$
\left\{\begin{array}{l}
n_{1}=L_{1} \\
n_{i}=L_{i}-L_{i-1}
\end{array}\right.
$$

where, in the bond graph model in preferential integral causality, $L_{i}$ is the smallest sum of the orders of $i$ different I/O causal paths.

Note. This procedure enables of increasingly ordering the infinite zero orders.

\section{Bond graph approach: Bicausal procedure}

In this subsection, a bicausal procedure to determine the minors of the system matrix of the inverse model is deduced from procedure 2 . After that, a procedure will be proposed to determine the highest degree $\delta_{i}$ of the minors of order $i$ of $\mathbf{T}_{\text {inv }}(s)$ on a bicausal bond graph model. Finally, a bicausal procedure to compute the infinite pole orders will be deduced.

Procedure 4. In a bicausal bond graph model, the minors of the system matrix $\mathbf{P}_{\mathbf{i n v}}(s)$ are given by the following expression:

$$
\begin{aligned}
& \operatorname{det}\left(\mathbf{P}_{\mathbf{i n v}}(s)_{1, \ldots, n_{i n v}, n_{i n v}+j_{1}, \ldots, n_{i n v}+j_{i}}^{1, \ldots, n_{i n v}}\right)= \\
& \sum_{k=-n_{D}}^{n_{i n v}} \rho_{k}^{\left\{i_{h_{1}, \ldots, h_{i}}^{j_{1}, \ldots, j_{i}}\right\}} s^{n_{i n v}-k}
\end{aligned}
$$


where:

- $n_{\text {inv }}\left(\right.$ resp. $\left.n_{D}\right)$ corresponds to the number of storage elements in integral causality (resp. in derivative causality) and $m$ is the number of inputs/outputs;

- $\rho_{k}^{\left\{i_{h_{1}, \ldots, h_{i}}^{j_{1}, \ldots, j_{i}}\right\}}$ corresponds to the sum of the static gains of bond graph families of order $k$ and containing only $i$ different I/O causal paths between $y_{j_{1}}, \ldots, y_{j_{i}}$ and $u_{h_{1}}, \ldots, u_{h_{i}}$. In this calculation, the static gain of each bond graph family must be multiplied by $(-1)^{d}$ where $d$ is the number of disjoint cycles contained in the bond graph family such $d \geq m$. The static gain of each family must be multiplied also by $(-1)^{\sigma_{k}}$ where $\sigma_{k}$ is the number of permutations needed to order the inputs $u_{h_{1}}, \ldots, u_{h_{i}}$ in the initial order of the input vector when the i I/O causal paths of the family are ordered in the initial order of the output vector.

Note. $\operatorname{det}\left(\mathbf{P}_{\mathbf{i n v}}(s)_{1, \ldots, n_{i n v}, n_{i n v}+h_{1}, \ldots, n_{i n v}+h_{i}}^{1, \ldots, n_{i n v}}\right)$ is computed from the bicausal bond graph model where only the double sources associated to $y_{j_{1}}, \ldots, y_{j_{i}}$ and the double detectors associated to $u_{i_{1}}, \ldots, u_{i_{i}}$ are considered in this calculation ${ }^{5}$.

Now, let us describe a bond graph procedure to determine graphically the highest degree of the minors of order $i$ of $\mathbf{T}_{\text {inv }}(s)$.

Procedure 5. Let $\delta_{i}$ be the highest degree of the minors of order $i$ of $\mathbf{T}_{\mathbf{i n v}}(s)$, then

$$
\forall i \in\{1 \ldots m\}, \delta_{i}=-L_{i}
$$

where, in the bicausal bond graph model, $L_{i}$ is the smallest order that a set of $i$ different I/O causal paths can have.

Proof. Let $\delta_{i}$ be the highest degree of the minors of order $i$ of $\mathbf{T}_{\mathbf{i n v}}(s)$, and $\beta_{i}$ be the highest degree of the minors of order $i$ of $\mathbf{P}_{\text {inv }}(s)$, and let $L_{i}$ be the smallest order that a set of $i$ different $\mathrm{I} / \mathrm{O}$ causal paths can have on the bicausal bond graph model. From Theorem 2 it can be deduced that $\delta_{i}=\beta_{i}-n_{i n v} . n_{i n v}$ is the number of storage elements in integral causality on the bicausal bond graph model. Each $\beta_{i}$ can be computed as follows:

$$
\beta_{i}=\max _{\substack{j_{1}, \ldots, j_{i} \in(\llbracket 1, m \rrbracket)^{i} \\ h_{1}, \ldots, h_{i} \in(\llbracket 1, m \rrbracket)^{i}}}\left\{\beta_{i_{h_{1}, \ldots, h_{i}}^{j_{1}, \ldots, j_{i}}}\right\}
$$

where:

$\beta_{i_{h_{1}}^{j_{1}, \ldots, h_{i}}}=\operatorname{deg}\left(\operatorname{det}\left(\mathbf{P}_{\mathbf{i n v}}(s)_{1, \ldots, n_{i n v}, n_{i n v}+h_{1}, \ldots, n_{i n v}+h_{i}}^{1, \ldots, n_{i n v}, n_{i n v}+j_{1}, \ldots, n_{i n v}+j_{i}}\right)\right)$.

As $\operatorname{det}\left(\mathbf{P}_{\mathbf{i n v}}(s)_{1, \ldots, n_{i n v}, n_{i n v}+h_{1}, \ldots, n_{i n v}+h_{i}}^{1, \ldots}\right) \neq 0$, then from procedure 4:

$$
\begin{gathered}
\operatorname{det}\left(\mathbf{P}_{\mathbf{i n v}}(s)_{1, \ldots, n_{i n v}, n_{i n v}+j_{1}, \ldots, n_{i n v}+n_{1}, \ldots, n_{i n v}+h_{i}}^{1, \ldots, n_{i}}\right)= \\
\sum_{k=-n_{D}}^{n_{i n v}} \rho_{k}^{\left\{i_{h_{1}, \ldots, h_{i}}^{j_{1}, \ldots, j_{i}}\right\}_{s}} s^{n_{i n v}-k}
\end{gathered}
$$

\footnotetext{
${ }^{5}$ The other double sources and double detectors are not deleted from the bicausal bond graph model in order not to change the causality assignment.
}

So:

$$
\begin{aligned}
& \operatorname{det}\left(\mathbf{P}_{\mathbf{i n v}}(s)_{1, \ldots, n_{i n v}, n_{i n v}+h_{1}, \ldots, n_{i n v}+h_{i}}^{1, \ldots, n_{i n}, n_{i n v}+j_{1}, \ldots, n_{i n}+j_{i}} \underset{+\infty}{\sim}\right.
\end{aligned}
$$

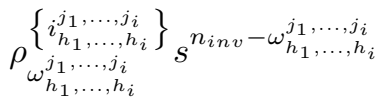

where $\omega_{h_{1}, \ldots, h_{i}}^{j_{1}, \ldots, j_{i}}$ is the smallest order that a bond graph family can have (this family contains exactly $i$ different I/O causal paths between $y_{j_{1}}, \ldots, y_{j_{i}}$ and $u_{h_{1}}, \ldots, u_{h_{i}}$ ).

In this paper, we consider only the structural analysis ${ }^{6}$, so $\rho_{\omega_{h_{1}, \ldots, h_{i}}^{j_{1}, \ldots, h_{i}}}^{\left\{\begin{array}{l}\left.i_{h_{1}}^{j_{1}, \ldots, h_{i}}\right\} \\ j_{1}\end{array}\right.}$ is supposed not equal to 0 . In this case:

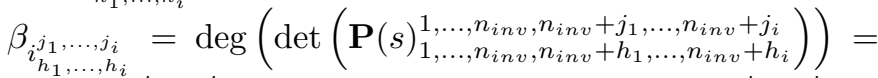
$n_{i n v}-\omega_{h_{1}, \ldots, h_{i}}^{j_{1}, \ldots, j_{i}}$ and consequently: $\delta_{i_{h_{1}, \ldots, h_{i}}^{j_{1}, \ldots, j_{i}}}=-\omega_{h_{1}, \ldots, h_{i}}^{j_{1}, \ldots, j_{i}}=$ $-L_{i}$.

End of proof.

From procedure 5, a bond graph procedure to obtain graphically the orders of the infinite poles of the inverse model (4) will be deduced and shown.

Procedure 6. Let us consider a bond graph model representing the inverse model (3). The number of infinite poles is equal to $m$ and each infinite pole order $n_{i}$ can be computed as follows:

$$
\left\{\begin{array}{l}
n_{1}=-L_{1} \\
n_{i}=L_{i-1}-L_{i}
\end{array}\right.
$$

where, on the bicausal bond graph model, $L_{i}$ is the smallest sum of the orders of $i$ different I/O causal paths.

\section{Proof.}

- For $i=1$, from Theorem 1, we have: $n_{1}=\delta_{1}$. Moreover, it has been shown in procedure 5 that $\delta_{i}=-L_{i}$. So, the result $n_{1}=-L_{1}$ is immediately deduced.

- For $i \geq 2$ and from Theorem 1 and procedure 5:

$$
\begin{aligned}
n_{i} & =-\delta_{i-1}+\delta_{i} \\
& =L_{i-1}-L_{i}
\end{aligned}
$$

\section{End of proof.}

Note. This procedure enables, according to Theorem 1, to decreasingly order the infinite pole orders.

$$
\begin{aligned}
& { }^{6} \text { If we consider a deeper analysis level, } \rho \underset{\omega^{j_{1}, \ldots, j_{i}}}{\left\{i_{h_{1}, \ldots, h_{i}}^{j_{1}, \ldots, j_{i}}\right\}} \text { can be equal to zero. } \\
& \omega_{h_{1}, \ldots, h_{i}}^{j_{1}, \ldots, j_{i}} \\
& \text { In this case, the bond graph families of order } \omega_{h_{1}, \ldots, h_{i}}^{j_{1}, \ldots,,_{i}}+1 \text { must be considered }
\end{aligned}
$$

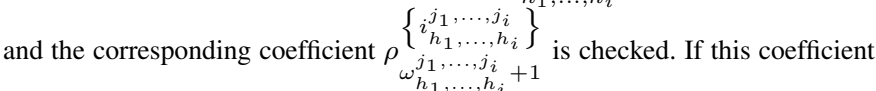

$$
\begin{aligned}
& \text { is equal to zero then the order } \omega_{h_{1}, \ldots, h_{i}}^{j_{1}, \ldots,,_{i}} \text { must be recurrently increased by } 1
\end{aligned}
$$

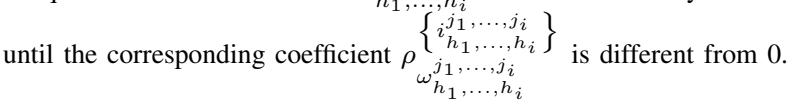




\section{Example}

In order to illustrate the two last procedures, let us consider the example composed of three masses in translation (Fig. 7). The connection between the masses $m_{1}$ and $m_{2}$ is modelled by means of a spring and a shock absorber in parallel. The connection between the masses $m_{2}$ and $m_{3}$ is modelled by means of a spring in parallel to an actuator delivering a force $F_{2}$. An effort $F_{1}$ is imposed on the mass $m_{1}$. The velocities $v_{2}$ and $v_{3}$ of the masses $m_{2}$ and $m_{3}$ are measured.

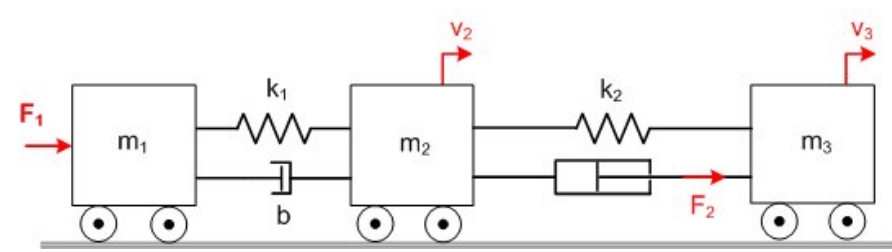

Fig. 7. Technological diagram of a mechanical system.

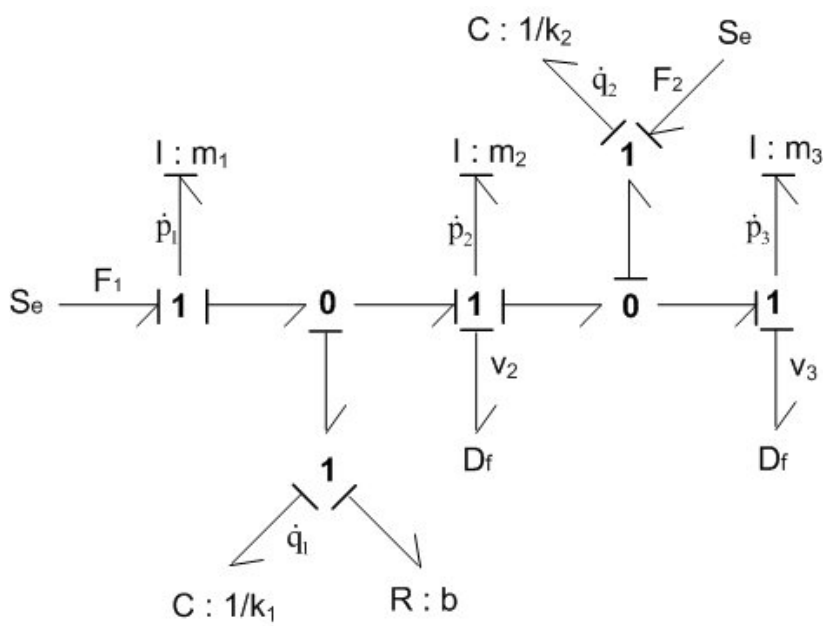

Fig. 8. Bond graph model of the mechanical system in preferential integral causality

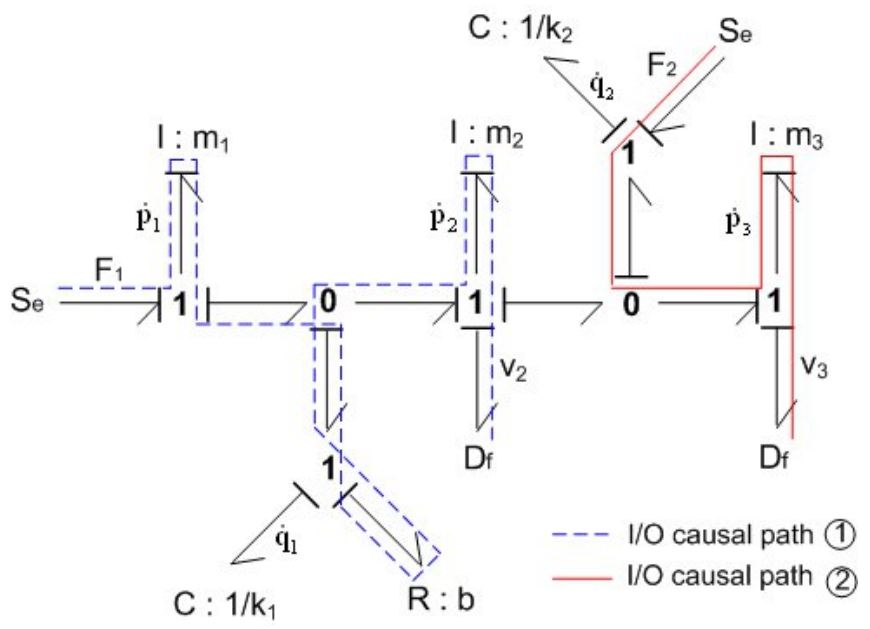

Fig. 9. Set $S_{1}$ of different I/O causal paths.

According to the rules of bond graph modelling, the associated bond graph model can be constructed. The preferential integral causality is assigned according to the SCAP procedure (i.e. Sequential Causality Assignment Procedure) [34] as shown in Fig. 8. From the causal bond graph model, the state-space model $\Sigma$ can be deduced with: $\mathbf{x}=\left[\begin{array}{lllll}p_{1} & p_{2} & p_{3} & q_{1} & q_{2}\end{array}\right]^{T}, \mathbf{u}=\left[\begin{array}{ll}F_{1} & F_{2}\end{array}\right]^{T}, \mathbf{y}=$ $\left[\begin{array}{ll}v_{2} & v_{3}\end{array}\right]^{T}, \quad \mathbf{A}=\left[\begin{array}{ccccc}-\frac{b}{m_{1}} & \frac{b}{m_{2}} & 0 & -k_{1} & 0 \\ \frac{b}{m_{1}} & -\frac{b}{m_{2}} & 0 & k_{1} & -k_{2} \\ 0 & 0 & 0 & 0 & k_{2} \\ \frac{1}{m_{1}} & -\frac{1}{m_{2}} & 0 & 0 & 0 \\ 0 & \frac{1}{m_{2}} & -\frac{1}{m_{3}} & 0 & 0\end{array}\right]$, $\mathbf{B}=\left[\begin{array}{cc}1 & 0 \\ 0 & 1 \\ 0 & -1 \\ 0 & 0 \\ 0 & 0\end{array}\right], \mathbf{C}=\left[\begin{array}{ccccc}0 & \frac{1}{m_{2}} & 0 & 0 & 0 \\ 0 & 0 & \frac{1}{m_{3}} & 0 & 0\end{array}\right]$ and $\mathbf{D}=[0]$

The transfer matrix $\mathbf{T}(s)$ of the direct model is written:

$$
\mathbf{T}(s)=\frac{1}{\operatorname{det}}\left[\begin{array}{ll}
t_{11} & t_{12} \\
t_{21} & t_{22}
\end{array}\right]
$$

with:

- $\operatorname{det}=s^{5}+b\left(\frac{1}{m_{1}}+\frac{1}{m_{2}}\right) s^{4}+\left(\frac{k_{1}}{m_{1}}+\frac{k_{1}+k_{2}}{m_{2}}+\frac{k_{2}}{m_{3}}\right) s^{3}+$ $b k_{2}\left(\frac{1}{m_{1} m_{2}}+\frac{1}{m_{2} m_{3}}+\frac{1}{m_{1} m_{3}}\right) s^{2}+k_{1} k_{2}\left(\frac{1}{m_{1} m_{2}}+\frac{1}{m_{2} m_{3}}+\right.$ $\left.\frac{1}{m_{1} m_{3}}\right) s$

- $t_{11}=\frac{b}{m_{1} m_{2}} s^{3}+\frac{k_{1}}{m_{1} m_{2}} s^{2}+\frac{b k_{2}}{m_{1} m_{2} m_{3}} s+\frac{k_{1} k_{2}}{m_{1} m_{2} m_{3}}$

- $t_{12}=\frac{1}{m_{2}} s^{4}+\frac{b}{m_{1} m_{2}} s^{3}+\frac{k_{1}}{m_{1} m_{2}} s^{2}$

- $t_{21}=\frac{b k_{2}}{m_{1} m_{2} m_{3}} s+\frac{k_{1} k_{2}}{m_{1} m_{2} m_{3}}$

- $t_{22}=-\frac{1}{m_{3}} s^{4}-\frac{b}{m_{3}}\left(\frac{1}{m_{1}}+\frac{1}{m_{2}}\right) s^{3}-\frac{k_{1}}{m_{3}}\left(\frac{1}{m_{1}}+\frac{1}{m_{2}}\right) s^{2}$

Using Theorem 1 gives the infinite zero orders by the statespace approach:

$$
\left\{\begin{array}{l}
t_{1}=\delta_{1}=-1 \\
t_{2}=\delta_{2}-\delta_{1}=-3+1=-2
\end{array}\right.
$$

So $n_{1}=1$ and $n_{2}=2$. This represents the reference result to which the bond graph-based one will be compared.

To determine the infinite zero orders from the bond graph model in preferential integral causality, the analysis of I/O causal paths is conducted (Fig. 9): $S_{1}=\{(1),(2)\}$ is the set of different I/O causal paths between the pair of inputs $\left(F_{1}, F_{2}\right)$ and the pair of outputs $\left(v_{2}, v_{3}\right)$ which has the smallest order ${ }^{7}$. So:

$$
\left\{\begin{array}{l}
L_{1}=\omega_{2}\left(F_{2} \rightarrow v_{3}\right)=1 \\
L_{2}=\omega_{1}\left(F_{1} \rightarrow v_{2}\right)+\omega_{2}\left(F_{2} \rightarrow v_{3}\right)=2+1=3
\end{array}\right.
$$

Then, it can be deduced that:

$$
\left\{\begin{array}{l}
n_{1}=L_{1}=1 \\
n_{2}=L_{2}-L_{1}=3-1=2
\end{array}\right.
$$

This result is in agreement with that found with the statespace approach, Theorem 1.

Now let us determine the infinite pole orders according firstly to the state-space approach, Theorem 1, and secondly

${ }^{7}$ The order of a set of I/O causal paths is the sum of the orders of all I/O causal paths of this set. 
according to the bicausal approach, procedure 6 .

The invertibility conditions are verified (for more details, see [29], [28]), so the bicausal bond graph model can be constructed as shown in Fig. 10 according to the SCAPI procedure (i.e. Sequential Causality Assignment Procedure for Inversion). From the bicausal bond graph model, the inverse model of minimal order (3) can be deduced:

$$
\left\{\begin{aligned}
\dot{\mathbf{q}}_{\mathbf{1}}= & -\frac{k_{1}}{b} \mathbf{q}_{\mathbf{1}}+\frac{m_{2}}{b} \dot{\mathbf{v}}_{\mathbf{2}}+\frac{m_{3}}{b} \dot{\mathbf{v}}_{\mathbf{3}} \\
\dot{\mathbf{q}}_{\mathbf{2}}= & \mathbf{v}_{\mathbf{2}}-\mathbf{v}_{\mathbf{3}} \\
\mathbf{F}_{\mathbf{1}}= & \frac{m_{1} k_{1}^{2}}{b^{2}} \mathbf{q}_{\mathbf{1}}+\frac{m_{1} m_{2}}{b} \ddot{\mathbf{v}}_{\mathbf{2}}+\frac{m_{1} m_{3}}{b} \ddot{\mathbf{v}}_{\mathbf{3}} \\
& +\left(m_{1}+m_{2}-\frac{m_{1} m_{2} k_{1}}{b^{2}}\right) \dot{\mathbf{v}}_{\mathbf{2}} \\
& +m_{3}\left(1-\frac{m_{1} k_{1}}{b^{2}}\right) \dot{\mathbf{v}}_{\mathbf{3}} \\
\mathbf{F}_{\mathbf{2}}= & k_{2} \mathbf{q}_{\mathbf{2}}-m_{3} \dot{\mathbf{v}}_{\mathbf{3}}
\end{aligned}\right.
$$

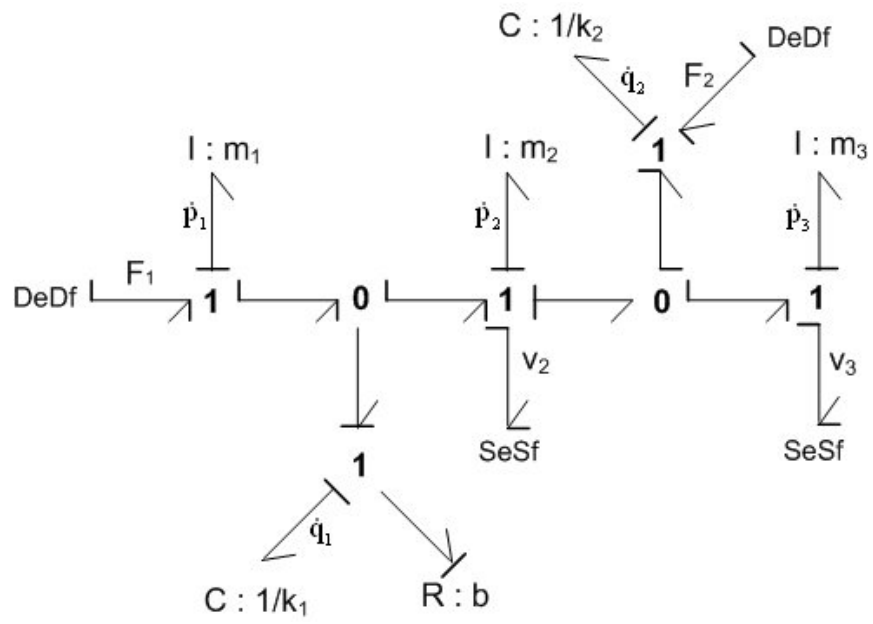

Fig. 10. Bicausal bond graph model of the mechanical system.

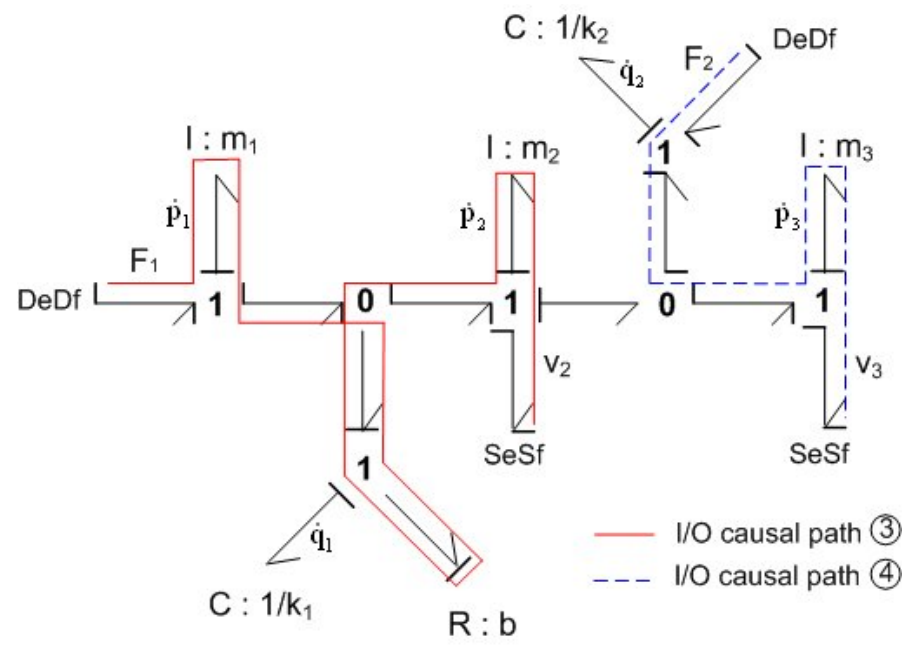

Fig. 11. Set $S_{2}$ of different I/O causal paths.

The transfer matrix $\mathbf{T}_{\mathbf{i n v}}(s)$ of the inverse model is written (34). According to Theorem 1, the infinite pole orders are computed by the state-space approach as follows:

$$
\left\{\begin{array}{l}
n_{1}=t_{1}=\delta_{1}=2 \\
n_{2}=t_{2}=\delta_{2}-\delta_{1}=3-2=1
\end{array}\right.
$$

This represents the reference result to which the bond graph-based one will be compared.

To determine the infinite pole orders from the bicausal bond graph model, the analysis of I/O causal paths is conducted (Fig. 11): $S_{2}=\{(3),(4)\}$ is the set of different I/O causal paths between the pair $\left(v_{2}, v_{3}\right)$ and the pair $\left(F_{1}, F_{2}\right)$ which has the smallest order. So:

$$
\left\{\begin{array}{l}
L_{1}=\omega_{3}\left(v_{2} \rightarrow F_{1}\right)=-2 \\
L_{2}=\omega_{3}\left(v_{2} \rightarrow F_{1}\right)+\omega_{4}\left(v_{3} \rightarrow F_{2}\right)=-2-1=-3
\end{array}\right.
$$

Then, it can be deduced that:

$$
\left\{\begin{array}{l}
n_{1}=-L_{1}=2 \\
n_{2}=L_{1}-L_{2}=-2+3=1
\end{array}\right.
$$

This result is in agreement with that found according to the state-space approach, Theorem 1.

\section{E. Interpretation and duality}

The two procedures (causal and bicausal procedures) enable of finding the same results as those found by the the state-space approach. The causal procedure 3 enables of determining the infinite zero orders from the bond graph model in preferential integral causality by the analysis of the orders of different $\mathrm{I} / \mathrm{O}$ causal paths and the bicausal procedure 6 enables of determining the infinite pole orders from the bicausal bond graph model by the analysis of the orders of different I/O causal paths. So the only difference between the two procedures (except the fact that the causal procedure is applied to the direct bond graph model and the bicausal procedure is applied to the inverse bond graph model) is that in the causal bond graph model the orders of I/O causal paths are considered and in the bicausal bond graph model the orders of I/O causal paths are considered with a minus sign.

\section{ESSENTIAL ORDER AND RELATIVE ORDER}

\section{A. State-space approach}

In this subsection, the state-space definitions of the relative order and the essential order are recalled. These definitions enable the computing of these orders from the direct model $\Sigma$. A new procedure to compute the relative orders from the inverse model (3) is proposed. This procedure will be used to demonstrate the bicausal bond graph procedure.

Theorem 3. The relative order $n_{i}^{\prime}$ of the output $y_{i}$ is to the minimal number of times it is necessary to time-differentiate the output $y_{i}$ in order to make explicitly appear at least one component of the input vector $\mathbf{u}$ in the expression of $y_{i}^{\left(n_{i}^{\prime}\right)}$. Such an order can be computed by one of these three following methods:

- $n_{i}^{\prime}$ is equal to the minimal difference between the polynomial degrees of the denominators and the numerators 


$$
\mathbf{T}_{\text {inv }}(s)=\left[\begin{array}{cc}
\frac{m_{1} m_{2} k_{1}^{2} s^{2}}{b^{3} s\left(s+\frac{k_{1}}{b}\right)}+\frac{m_{1} m_{2}}{b} s^{2}+\left(m_{1}+m_{2}-\frac{m_{1} m_{2} k_{1}}{b^{2}}\right) s & \frac{m_{1} m_{3} k_{1}^{2} s^{2}}{b^{3} s\left(s+\frac{k_{1}}{b}\right)}+\frac{m_{1} m_{3}}{b} s^{2}+\left(m_{3}-\frac{m_{1} m_{3} k_{1}}{b^{2}}\right) s \\
\frac{k_{2}\left(s+\frac{k_{1}}{b}\right)}{s\left(s+\frac{k_{1}}{b}\right)} & -\frac{k_{2}\left(s+\frac{k_{1}}{b}\right)}{s\left(s+\frac{k_{1}}{b}\right)}-m_{3} s
\end{array}\right]
$$

composing the transmittances of the I/O transfer matrix row $\mathbf{t}_{i}(s)$ [35], [30].

- $n_{i}^{\prime}$ can be calculated as [35], [36], [2]:

$$
n_{i}^{\prime}=\left\{\begin{array}{llc}
0 & \text { if } & \mathbf{d}_{i} \neq \mathbf{0} \\
\inf _{k \in \mathbb{N}^{*}}\{k & \mid & \left.\mathbf{c}_{i} \mathbf{A}^{k-1} \mathbf{B} \neq \mathbf{0}\right\}
\end{array}\right.
$$

- $n_{i}^{\prime}$ is equal to the order of the infinite zero of $\left(\mathbf{A}, \mathbf{B}, \mathbf{c}_{i}, \mathbf{d}_{i}\right)$, namely [35], [36]:

$$
n_{i}^{\prime}=\inf _{k \in \mathbb{N}}\left\{k \mid \lim _{s \rightarrow \infty} s^{k} \mathbf{t}_{i}(s) \neq \mathbf{0}\right\}
$$

where $\mathbf{c}_{i}\left(\right.$ resp. $\left.\mathbf{d}_{i}\right)$ is the $i^{\text {th }}$ row of $\mathbf{C}($ resp. $\mathbf{D})$ and $\mathbf{t}_{i}(s)=$ $\mathbf{c}_{i}(s \mathbf{I}-\mathbf{A})^{-1} \mathbf{B}+\mathbf{d}_{i}$.

Theorem 4. The essential order $n_{i e}$ of the output $y_{i}$ is equal to the highest time-differentiation order of the output $y_{i}$ appearing in the inverse model [2], [22]. For a right-invertible system $(\mathbf{A}, \mathbf{B}, \mathbf{C})$, the essential order $n_{i e}$ of the output $y_{i}$ can be expressed as [2]:

$$
n_{i e}=\sum_{j=1}^{m} n_{j}-\sum_{j=1}^{m-1} \bar{n}_{i j}
$$

where:

- $n_{j}$ is the $j^{\text {th }}$ infinite zero order of the system $(\mathbf{A}, \mathbf{B}, \mathbf{C})$;

- $\bar{n}_{i j}$ is the $j^{\text {th }}$ infinite zero order of the system $\left(\mathbf{A}, \mathbf{B}, \overline{\mathbf{C}}_{i}\right)$ with:

$$
\overline{\mathbf{C}}_{i}=\left[\begin{array}{lllllll}
c_{1}^{T} & c_{2}^{T} & \ldots & c_{i-1}^{T} & c_{i+1}^{T} & \ldots & c_{m}^{T}
\end{array}\right]^{T}
$$

and $\forall k \in\{1, \ldots, i-1, i+1, \ldots, m\}, c_{k}$ is the $k^{\text {th }}$ row of $\mathbf{C}$.

Now, let us propose a state-space procedure to compute the relative orders from the inverse model (4). This procedure will be used to display the duality between these orders and the essential orders.

Theorem 5. For a square invertible system, the relative order $n_{i}^{\prime}$ of the output $y_{i}$ can be expressed as:

$$
n_{i}^{\prime}=\sum_{j=1}^{m} n_{j}-\sum_{j=1}^{m-1} \bar{n}_{i j}
$$

With:

- $n_{j}$ is the $j^{\text {th }}$ infinite pole order of the system $\left(\mathbf{A}_{\text {inv }}, \mathbf{B}_{\mathbf{i n v}}(s), \mathbf{C}_{\mathbf{i n v}}, \mathbf{D}_{\mathbf{i n v}}(s)\right)$.

- $\bar{n}_{i j}$ is the $j^{\text {th }}$ infinite pole order of the system $\left(\mathbf{A}_{\mathbf{i n v}}, \overline{\mathbf{B}}_{\mathbf{i n v}_{\mathbf{i}}}(s), \mathbf{C}_{\mathbf{i n v}}, \overline{\mathbf{D}}_{\mathbf{i n v}_{\mathbf{i}}}(s)\right)$ with:

$\overline{\mathbf{B}}_{\mathbf{i n v}_{\mathbf{i}}}(s)=\left[\begin{array}{llllll}b_{i n v_{1}} & \ldots & b_{i n v_{i-1}} & b_{i n v_{i+1}} & \ldots & b_{i n v_{m}}\end{array}\right]$ $\overline{\mathbf{D}}_{\mathbf{i n v}_{\mathbf{i}}}(s)=\left[\begin{array}{llllll}d_{i n v_{1}} & \ldots & d_{i n v_{i-1}} & d_{i n v_{i+1}} & \ldots & d_{i n v_{m}}\end{array}\right]$ and $\forall k \in\{1, \ldots, i-1, i+1, \ldots, m\}, b_{\text {inv }_{k}}$ (resp. $d_{\text {inv }_{k}}$ ) is the $k^{\text {th }}$ column of $\mathbf{B}_{\mathbf{i n v}}(s)$ (resp. $\mathbf{D}_{\mathbf{i n v}}(s)$ ).
Proof. ${ }^{8}$ The transfer matrix of the inverse model $\mathbf{T}_{\mathbf{i n v}}(s)$ can be factorized as:

$$
\mathbf{T}_{\mathbf{i n v}}(s)=\left[\mathbf{T}_{\mathbf{i n v} \mathbf{1}}(s)\left|t_{i n v_{i}}(s)\right| \mathbf{T}_{\mathbf{i n v}_{\mathbf{2}}}(s)\right]
$$

with $t_{\text {inv }}(s)$ the $i^{\text {th }}$ column of $\mathbf{T}_{\mathbf{i n v}}(s)$.

The first step of this proof consists of a column permutation to simplify the manipulations of the matrix, thus:

$$
\begin{aligned}
\overline{\mathbf{T}}_{\mathbf{i n v}}(s) & =\mathbf{T}_{\mathbf{i n v}}(s) \mathbf{M} \\
& =\left[t_{i n v_{i}}(s)\left|\mathbf{T}_{\mathbf{i n v}_{\mathbf{1}}}(s)\right| \mathbf{T}_{\mathbf{i n v}_{\mathbf{2}}}(s)\right]
\end{aligned}
$$

with $\mathbf{M}$ the permutation matrix defined by:

$$
\mathbf{M}=\left[\begin{array}{c|ccc|ccc}
0 & 1 & & & 0 & \cdots & 0 \\
\vdots & & \ddots & & \vdots & \ddots & \vdots \\
0 & & & 1 & 0 & \cdots & 0 \\
\hline 1 & 0 & \cdots & 0 & 0 & \cdots & 0 \\
\hline 0 & 0 & \cdots & 0 & 1 & & \\
\vdots & \vdots & \ddots & \vdots & & \ddots & \\
0 & 0 & \cdots & 0 & & & 1
\end{array}\right]
$$

Thus $\overline{\mathbf{T}}_{\mathbf{i n v}}(s)$ and $\mathbf{T}_{\mathbf{i n v}}(s)$ have the same structure at infinity and the infinite pole order of the first column of $\overline{\mathbf{T}}_{\mathbf{i n v}}(s)$ is equal to the infinite pole order of the $i^{t h}$ column of $\mathbf{T}_{\mathbf{i n v}}(s)$. So the infinite zero order of the $i^{t h}$ row of $\mathbf{T}(s)=\mathbf{T}_{\mathbf{i n v}}^{-\mathbf{1}}(s)$ is equal to the infinite zero order of the first row of $\overline{\mathbf{T}}_{\mathbf{i n v}}^{-1}(s)$.

The second step of this proof is to express the relation $\overline{\mathbf{T}}_{\text {inv }}(s)$ and the Smith-McMillan factorization at infinity of the subsystem $\left(\mathbf{A}_{\mathbf{i n v}}, \overline{\mathbf{B}}_{\mathbf{i n v}_{\mathbf{i}}}(s), \mathbf{C}_{\mathbf{i n v}}, \overline{\mathbf{D}}_{\mathbf{i n v}_{\mathbf{i}}}(s)\right)$ where:

$$
\begin{aligned}
& \overline{\mathbf{B}}_{\mathbf{i n v}_{\mathbf{i}}}(s)=\left[\begin{array}{llllll}
b_{i n v_{1}} & \ldots & b_{i n v_{i-1}} & b_{i n v_{i+1}} & \ldots & b_{i n v_{m}}
\end{array}\right] \\
& \overline{\mathbf{D}}_{\mathbf{i n v}_{\mathbf{i}}}(s)=\left[\begin{array}{llllll}
d_{i n v_{1}} & \ldots & d_{i n v_{i-1}} & d_{i n v_{i+1}} & \ldots & d_{i n v_{m}}
\end{array}\right]
\end{aligned}
$$

and $\forall k \in\{1, \ldots, i-1, i+1, \ldots, m\}, b_{i n v_{k}}$ (resp. $d_{i n v_{k}}$ ) is the $k^{t h}$ column of $\mathbf{B}_{\mathbf{i n v}}(s)$ (resp. $\mathbf{D}_{\mathbf{i n v}}(s)$ ).

In fact, the Smith-McMillan factorization at infinity of the subsystem $\left(\mathbf{A}_{\mathbf{i n v}}, \overline{\mathbf{B}}_{\mathbf{i n v}_{\mathbf{i}}}(s), \mathbf{C}_{\mathbf{i n v}}, \overline{\mathbf{D}}_{\mathbf{i n v}_{\mathbf{i}}}(s)\right)$, characterized by the transfer matrix $\left[\mathbf{T}_{\mathbf{i n v}}(s) \mid \mathbf{T}_{\mathbf{i n v}}(s)\right]$, is defined by:

$$
\left[\mathbf{T}_{\mathbf{i n v}_{\mathbf{1}}}(s) \mid \mathbf{T}_{\mathbf{i n v}_{\mathbf{2}}}(s)\right]=\overline{\mathbf{B}}_{\mathbf{1}}(s) \overline{\boldsymbol{\Lambda}}(s) \overline{\mathbf{B}}_{\mathbf{2}}(s)
$$

with $\overline{\mathbf{B}}_{\mathbf{1}}(s)$ and $\overline{\mathbf{B}}_{\mathbf{2}}(s)$ biproper matrices of respective dimensions $(\mathrm{m} \times \mathrm{m})$ and $(\mathrm{m}-1 \times \mathrm{m}-1)$ and $\overline{\boldsymbol{\Lambda}}(s)=$ $\left[\frac{\overline{\boldsymbol{\Delta}}(s)}{0}\right]$, where $\overline{\boldsymbol{\Delta}}(s)=\operatorname{diag}\left(s^{\bar{n}_{i 1}}, \ldots, s^{\bar{n}_{i(m-1)}}\right)$. $\bar{n}_{i 1}, \ldots, \bar{n}_{i(m-1)}$ are the infinite pole orders of the system

${ }^{8}$ This proof is similar to the proof of the definition of essential order (Theorem 4) presented in [2]. The only difference is that this proof is applied to the inverse model (4) while the proof in [2] is applied to the direct model $\Sigma$. 
$\left(\mathbf{A}_{\mathbf{i n v}}, \overline{\mathbf{B}}_{\mathbf{i n v}_{\mathbf{i}}}(s), \mathbf{C}_{\mathbf{i n v}}, \overline{\mathbf{D}}_{\mathbf{i n v}_{\mathbf{i}}}(s)\right)$. So $\overline{\mathbf{T}}_{\mathbf{i n v}}(s)$ can be expressed as:

$$
\begin{aligned}
\overline{\mathbf{T}}_{\mathbf{i n v}}(s) & =\left[t_{i n v_{i}}(s) \mid \overline{\mathbf{B}}_{\mathbf{1}}(s) \overline{\boldsymbol{\Lambda}}(s) \overline{\mathbf{B}}_{\mathbf{2}}(s)\right] \\
& =\overline{\mathbf{B}}_{\mathbf{1}}(s)\left[\overline{\mathbf{B}}_{\mathbf{1}}^{-\mathbf{1}}(s) t_{i n v_{i}}(s) \mid \overline{\mathbf{\Lambda}}(s) \overline{\mathbf{B}}_{\mathbf{2}}(s)\right]
\end{aligned}
$$

Under the left action of biproper transformation, the structure at infinity of the columns of $\overline{\mathbf{T}}_{\mathbf{i n v}}(s)$ corresponds to that of $\left[\overline{\mathbf{B}}_{\mathbf{1}}^{-\mathbf{1}}(s) t_{\text {invi }}(s) \mid \overline{\boldsymbol{\Lambda}}(s) \overline{\mathbf{B}}_{\mathbf{2}}(s)\right]$. This result can be shown thanks to the properties of a biproper matrix and thanks to the following definition of the infinite pole order of a column $\mathbf{g}_{i}(s)$ [37]:

$$
\inf _{k \in \mathbb{N}^{*}}\left\{k \mid \lim _{s \rightarrow \infty} s^{-k} \mathbf{g}_{i}(s) \neq \mathbf{0}\right\}
$$

It can be deduced that, under the right action of the biproper matrix $\overline{\mathbf{B}}_{\mathbf{1}}^{-\mathbf{1}}(s)$, the structure at infinity of the rows of $\overline{\mathbf{T}}_{\mathbf{i n v}}^{-\mathbf{1}}(s)$ corresponds to that of $\left[\overline{\mathbf{B}}_{\mathbf{1}}^{-\mathbf{1}}(s) t_{\text {invi }_{i}}(s) \mid \overline{\mathbf{\Lambda}}(s) \overline{\mathbf{B}}_{\mathbf{2}}(s)\right]^{-1}$.

Moreover,

$$
\begin{aligned}
& {\left[\overline{\mathbf{B}}_{\mathbf{1}}^{-\mathbf{1}}(s) t_{i n v_{i}}(s) \mid \overline{\mathbf{\Lambda}}(s) \overline{\mathbf{B}}_{\mathbf{2}}(s)\right]} \\
& =\overline{\mathbf{T}}(s)\left[\begin{array}{c|ccc}
1 & 0 & \ldots & 0 \\
\hline 0 & & & \\
\vdots & & \overline{\mathbf{B}}_{\mathbf{2}}(s) & \\
0 & & &
\end{array}\right]
\end{aligned}
$$

with $\overline{\mathbf{T}}(s)=\left[\overline{\mathbf{B}}_{\mathbf{1}}^{-\mathbf{1}}(s) t_{\text {invi }_{i}}(s) \mid \overline{\mathbf{\Lambda}}(s)\right]$. Thus,

$$
\begin{aligned}
& {\left[\overline{\mathbf{B}}_{\mathbf{1}}^{-\mathbf{1}}(s) t_{\text {invi }_{i}}(s) \mid \overline{\boldsymbol{\Lambda}}(s) \overline{\mathbf{B}}_{\mathbf{2}}(s)\right]^{-1}=} \\
& {\left[\begin{array}{c|ccc}
1 & 0 & \ldots & 0 \\
\hline 0 & & \\
\vdots & & \overline{\mathbf{B}}_{\mathbf{2}}^{-1}(s) \\
0 & &
\end{array}\right] \overline{\mathbf{T}}^{-\mathbf{1}}(s)}
\end{aligned}
$$

Thanks to the particular form of the biproper matrix, the first row of $\left[\overline{\mathbf{B}}_{\mathbf{1}}^{-\mathbf{1}}(s) t_{i n v_{i}}(s) \mid \overline{\boldsymbol{\Lambda}}(s) \overline{\mathbf{B}}_{\mathbf{2}}(s)\right]^{-1}$ corresponds to the first row of $\overline{\mathbf{T}}^{-\mathbf{1}}(s)$. So, it can be deduced that the first rows of $\overline{\mathbf{T}}_{\mathbf{i n v}}^{-1}(s)$ and $\overline{\mathbf{T}}^{-1}(s)$ have the same infinite zero order and consequently, the $i^{t h}$ row of $\mathbf{T}(s)$ has this same infinite zero order.

Now, let us determine the first row of $\overline{\mathbf{T}}^{-1}(s)$. In fact, the inverse of this matrix is defined by:

$$
\overline{\mathbf{T}}^{-\mathbf{1}}(s)=\frac{1}{\operatorname{det}(\overline{\mathbf{T}}(s))} \operatorname{adj}(\overline{\mathbf{T}}(s))
$$

The first row of $\operatorname{adj}(\overline{\mathbf{T}}(s))$ is defined by:

$$
\left[\begin{array}{llll}
0 & \ldots & 0 & (-1)^{(m-1)}
\end{array} \prod_{j=1}^{m-1} s^{\bar{n}_{i j}}\right]
$$

Since

$$
\overline{\mathbf{T}}_{\mathbf{i n v}}(s)=\overline{\mathbf{B}}_{\mathbf{1}}(s) \overline{\mathbf{T}}(s)\left[\begin{array}{c|ccc}
1 & 0 & \ldots & 0 \\
\hline 0 & & \\
\vdots & & \overline{\mathbf{B}}_{\mathbf{2}}(s) \\
0 & &
\end{array}\right]
$$

Then,

$$
\overline{\mathbf{T}}(s)=\overline{\mathbf{B}}_{\mathbf{1}}^{-\mathbf{1}}(s) \overline{\mathbf{T}}_{\mathbf{i n v}}(s)\left[\begin{array}{c|ccc}
1 & 0 & \ldots & 0 \\
\hline 0 & & \\
\vdots & \overline{\mathbf{B}}_{\mathbf{2}}^{-1}(s) \\
0 & &
\end{array}\right]
$$

Also, the Smith-MacMillan factorization at infinity of $\mathbf{T}_{\mathbf{i n v}}(s)$ can be expressed as:

$$
\mathbf{T}_{\mathbf{i n v}}(s)=\mathbf{B}_{\mathbf{1}}(s) \boldsymbol{\Lambda}(s) \mathbf{B}_{\mathbf{2}}(s)
$$

with $\mathbf{B}_{\mathbf{1}}(s)$ and $\mathbf{B}_{\mathbf{2}}(s)$ biproper matrices and $\boldsymbol{\Lambda}(s)=$ $\operatorname{diag}\left(s^{n_{1}}, \ldots, s^{n_{m}}\right)$ where $\left\{n_{1}, \ldots, n_{m}\right\}$ are the orders of the poles at infinity of $\mathbf{T}_{\mathbf{i n v}}(\mathbf{s})$. Thus,

$\overline{\mathbf{T}}(s)=\overline{\mathbf{B}}_{\mathbf{1}}^{-\mathbf{1}}(s) \mathbf{B}_{\mathbf{1}}(s) \boldsymbol{\Lambda}(s) \mathbf{B}_{\mathbf{2}}(s) \mathbf{M}\left[\begin{array}{c|ccc}1 & 0 & \ldots & 0 \\ \hline 0 & & \\ \vdots & & \overline{\mathbf{B}}_{\mathbf{2}}^{-\mathbf{1}}(s) \\ 0 & \end{array}\right.$

The determinant of $\overline{\mathbf{T}}(s)$ is:

$$
\operatorname{det}(\overline{\mathbf{T}}(s))=b(s) \prod_{j=1}^{m} s^{n_{j}}
$$

with $b(s)$ a biproper function resulting from the determinants of the biproper matrices of the factorization at infinity of $\overline{\mathbf{T}}(s)$ (49). Thus the first row of $\overline{\mathbf{T}}^{-1}(s)$ is defined by:

$$
\left[\begin{array}{llll}
0 & \ldots & 0 & \frac{(-1)^{(m-1)} \prod_{j=1}^{m-1} s^{\bar{n}_{i j}}}{b(s)} \prod_{j=1}^{m} s^{n_{j}}
\end{array}\right]
$$

So, the infinite zero order of the first row of $\overline{\mathbf{T}}^{-\mathbf{1}}(\mathbf{s})$, and consequently of the $i^{\text {th }}$ row of $\mathbf{T}(\mathbf{s})$, corresponds to: $n_{i}^{\prime}=$ $-\left(\sum_{j=1}^{m-1} \bar{n}_{i j}-\sum_{j=1}^{m} n_{j}\right)$. The infinite zero order of the $i^{t h}$ row of $\mathbf{T}(\mathbf{s})$ is defined as the relative order of $y_{i}$. Then, the relative order associated to $y_{i}$ is defined by:

$$
n_{i}^{\prime}=\sum_{j=1}^{m} n_{j}-\sum_{j=1}^{m-1} \bar{n}_{i j}
$$

End of proof.

Theorem 5 will be used to define the bicausal procedure for the determination of the relative orders.

\section{B. Bond graph approach: Causal procedures}

The causal procedures are applied to the bond graph model in preferential integral causality to determine the relative orders and the essential orders from the direct model $\Sigma$.

Procedure 7. In a bond graph model in preferential integral causality, the relative order $n_{i}^{\prime}$ of the output $y_{i}$ is equal to the 
minimal order a causal path can have between the output $y_{i}$ and any input [19], [15] .

Procedure 8. In a bond graph model in preferential integral causality, the essential order $n_{i e}$ of the output $y_{i}$ can be computed as follows [38]:

$$
n_{i e}=L_{m}-L_{m-1}^{(i)}
$$

where:

- $L_{m}$ is the smallest sum of the orders of $m$ different I/O causal paths in the initial bond graph model;

- $L_{m-1}^{(i)}$ is the smallest sum of the orders of $m-1$ different I/O causal paths in the bond graph model obtained by deleting the $i^{\text {th }}$ detector element in the initial bond graph model.

\section{Bond Graph approach: Bicausal procedures}

The bicausal procedures are applied on bicausal bond graph model to determine the relative orders and the essential orders from the inverse model (3).

Procedure 9. In a bicausal bond graph model, the relative order $n_{i}^{\prime}$ of $y_{i}$ can be expressed as:

$$
n_{i}^{\prime}=L_{m-1}^{(i)}-L_{m}
$$

where:

- $L_{m}$ is the smallest sum of the orders of $m$ different $I / O$ causal paths in the bicausal bond graph model;

- $L_{m-1}^{(i)}$ is the smallest sum of the orders of $m-1$ different I/O causal paths obtained without considering, in the bicausal bond graph model, the $i^{\text {th }}$ double source element ${ }^{10}$.

Proof. From procedure 6 , it can be deduced that $\sum_{j=1}^{m} n_{j}=$ $-L_{m}$ and $\sum_{j=1}^{m-1} \bar{n}_{i j}=-L_{m-1}^{(i)}$. So, from these results and from Theorem 5, this bicausal procedure can be deduced. End of proof.

Procedure 10. In a bicausal bond graph model, the essential order $n_{i e}$ of $y_{i}$ can be expressed as [39], [38]:

$$
n_{i e}=-\min _{k, j}\left\{\omega_{k}\left(y_{i} \rightarrow u_{j}\right)\right\}
$$

where $\min _{k, j}\left\{\omega_{k}\left(y_{i} \rightarrow u_{j}\right)\right\}$ is the minimal order a causal path can have between the double source associated with $y_{i}$ and any double detector.

\footnotetext{
${ }^{9}$ Attention has to be paid to the fact that an exception to this definition may happen. As highlighted in [15], if there are two paths having the same minimal order $\omega_{i_{\text {min }}}$ and such that the sum of their gains is equal to zero, then the relative order of the studied output can be greater than $\omega_{i_{m i n}}$. Actually, only $n_{i}^{\prime} \geq \omega_{i_{\text {min }}}$ is true in the general case.

${ }^{10}$ The $i^{t h}$ double source element is not deleted from the bicausal bond graph model in order to not change the causality assignment.
}

\section{Example}

Let us consider the mechanical system illustrated in Fig. 7. The use of Theorem 3 and Theorem 4 enables to deduce the relative orders and the essential orders from the direct model $\Sigma$ by a state-space approach (26): $n_{1}^{\prime}=1$, $n_{2}^{\prime}=1, n_{1 e}=n_{1}+n_{2}-\bar{n}_{11}=1+2-1=2$ and $n_{2 e}=n_{1}+n_{2}-\bar{n}_{21}=1+2-1=2$. This represents the reference result to which the bond graph-based ones will be compared.

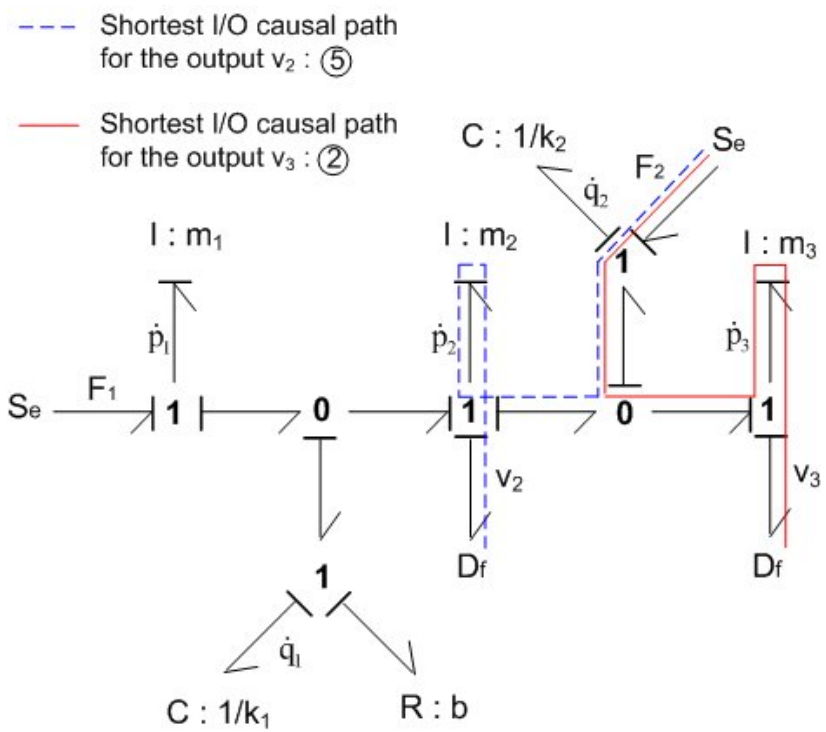

Fig. 12. Determination of the relative orders.

Now let us determine the relative orders and the essential orders by the bond graph causal procedures:

- The analysis of the bond graph model in preferential integral causality leads to the conclusion that the shortest causal path between the output $v_{2}$ (resp. $v_{3}$ ) and any input corresponds to the path (5) (resp. (2)) between $v_{2}$ (resp. $\left.v_{3}\right)$ and $F_{2}$ as shown in Fig. 12. So $n_{1}^{\prime}=\omega_{5}\left(F_{2} \rightarrow v_{2}\right)=$ 1 and $n_{2}^{\prime}=\omega_{2}\left(F_{2} \rightarrow v_{3}\right)=1$.

- To compute the essential orders, let us determine the infinite zero orders of the subsystem $\left(\mathbf{A}, \mathbf{B}, \overline{\mathbf{C}}_{\mathbf{1}}\right)$ (resp. $\left(\mathbf{A}, \mathbf{B}, \overline{\mathbf{C}}_{\mathbf{2}}\right)$ ). For that, let us consider the bond graph models shown in Fig. 13(a) and in Fig. 13(b) where the elements $D_{f}: v_{2}$ and $D_{f}: v_{3}$ have been respectively deleted from the initial bond graph model. From their causal analysis, it follows that:

$$
\left\{\begin{array}{l}
L_{2}=3 \text { Fig. } 9 \text { and }(28) \\
L_{1}^{(1)}=\omega_{2}\left(F_{2} \rightarrow v_{3}\right)=1 \\
L_{1}^{(2)}=\omega_{5}\left(F_{2} \rightarrow v_{2}\right)=1
\end{array}\right.
$$

So, according to procedure 8 , it can be concluded that:

$$
\left\{\begin{array}{l}
n_{1 e}=L_{2}-L_{1}^{(1)}=3-1=2 \\
n_{2 e}=L_{2}-L_{1}^{(2)}=3-1=2
\end{array}\right.
$$

These results are in agreement with those found using the state-space approach. 


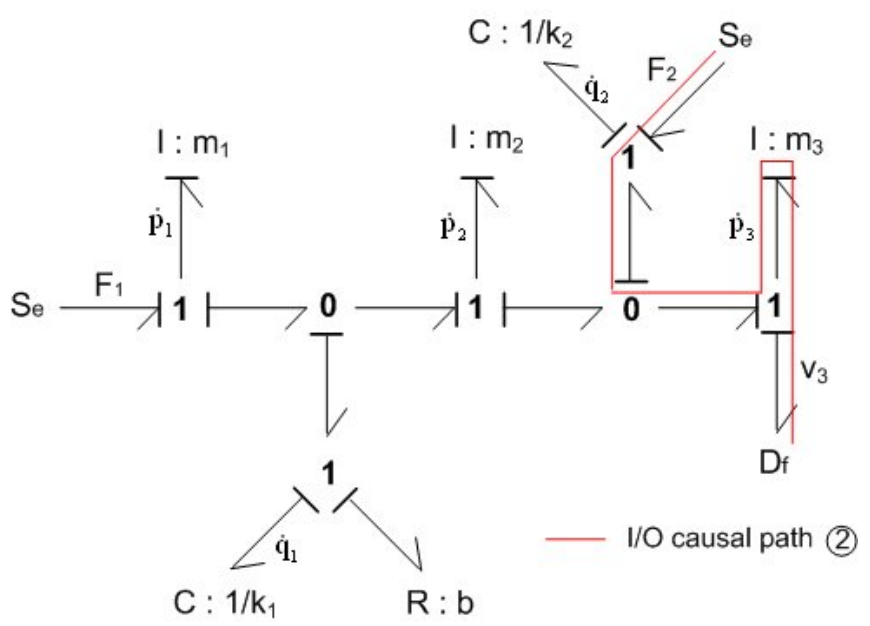

(a)

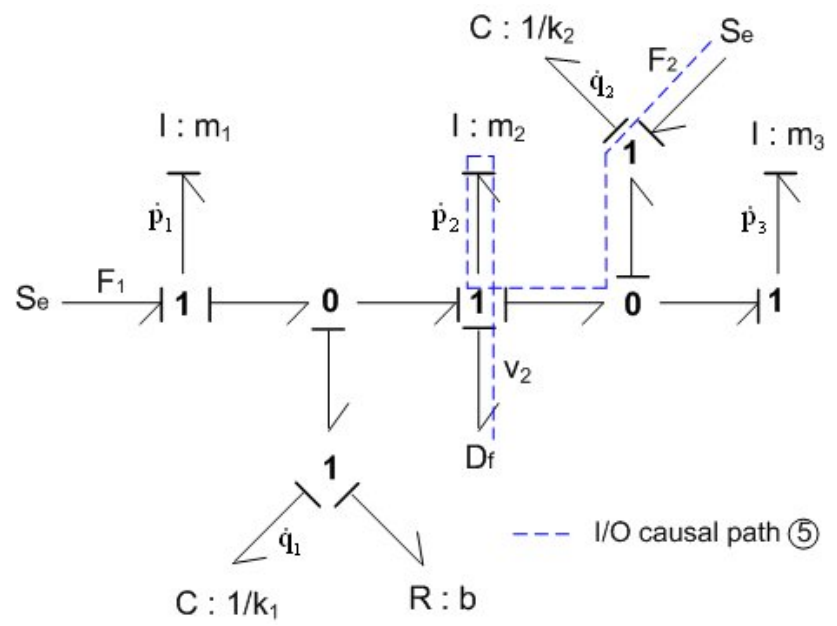

(b)

Fig. 13. Determination of infinite zero orders $\bar{n}_{i j}$.

Now, let us determine the relative orders and the essential orders by the bicausal procedures:

- To compute the relative orders, let us determine the infinite pole orders of the subsystem $\quad\left(\mathbf{A}_{\text {inv }}, \overline{\mathbf{B}}_{\mathbf{i n v}_{\mathbf{1}}}(s), \mathbf{C}_{\mathbf{i n v}}, \overline{\mathbf{D}}_{\mathbf{i n v}_{\mathbf{1}}}(s)\right) \quad$ (resp. $\left.\left(\mathbf{A}_{\mathbf{i n v}}, \overline{\mathbf{B}}_{\mathbf{i n v}_{\mathbf{2}}}(s), \mathbf{C}_{\mathbf{i n v}}, \overline{\mathbf{D}}_{\mathbf{i n v}_{\mathbf{2}}}(s)\right)\right)$. For that, let us consider the bond graph models shown in Fig. 14(a) and in Fig. 14(b) where the elements $S_{e} S_{f}: v_{2}$ and $S_{e} S_{f}: v_{3}$ are respectively not considered. From their causal analysis, it follows that:

$$
\left\{\begin{array}{l}
L_{2}=-3 \text { Fig. } 11 \text { and }(32) \\
L_{1}^{(1)}=\omega_{6}\left(v_{3} \rightarrow F_{1}\right)=-2 \\
L_{1}^{(2)}=\omega_{3}\left(v_{2} \rightarrow F_{1}\right)=-2
\end{array}\right.
$$

So, according to procedure 9 , it can be concluded that:

$$
\left\{\begin{array}{l}
n_{1}^{\prime}=L_{1}^{(1)}-L_{2}=-2+3=1 \\
n_{2}^{\prime}=L_{1}^{(2)}-L_{2}=-2+3=1
\end{array}\right.
$$

- The analysis of the bicausal bond graph model leads to the conclusion that the smallest order causal path between the output $v_{2}$ (resp. $v_{3}$ ) and any input corresponds to

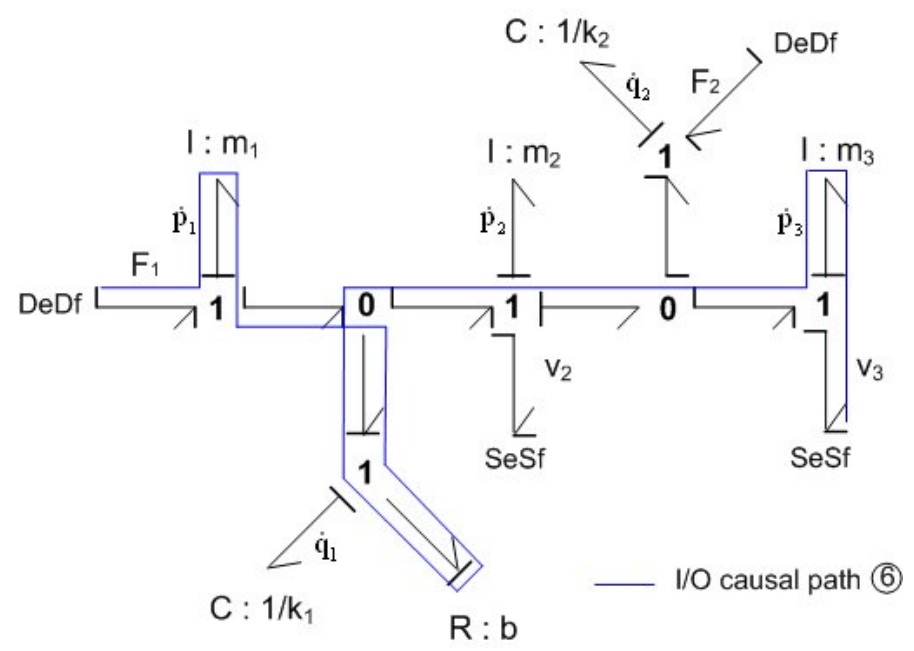

(a)

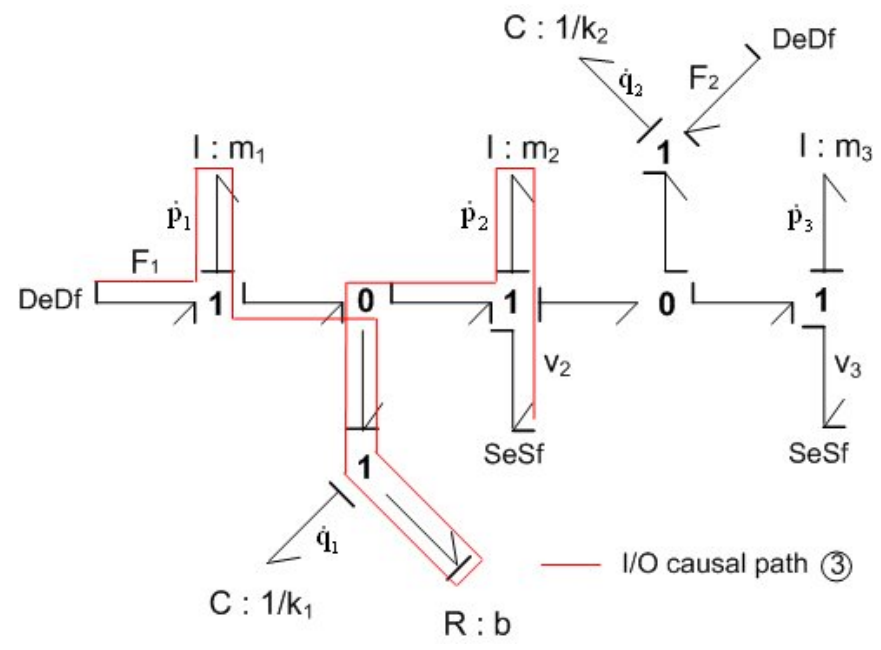

(b)

Fig. 14. Determination of infinite pole orders $\bar{n}_{i j}$.

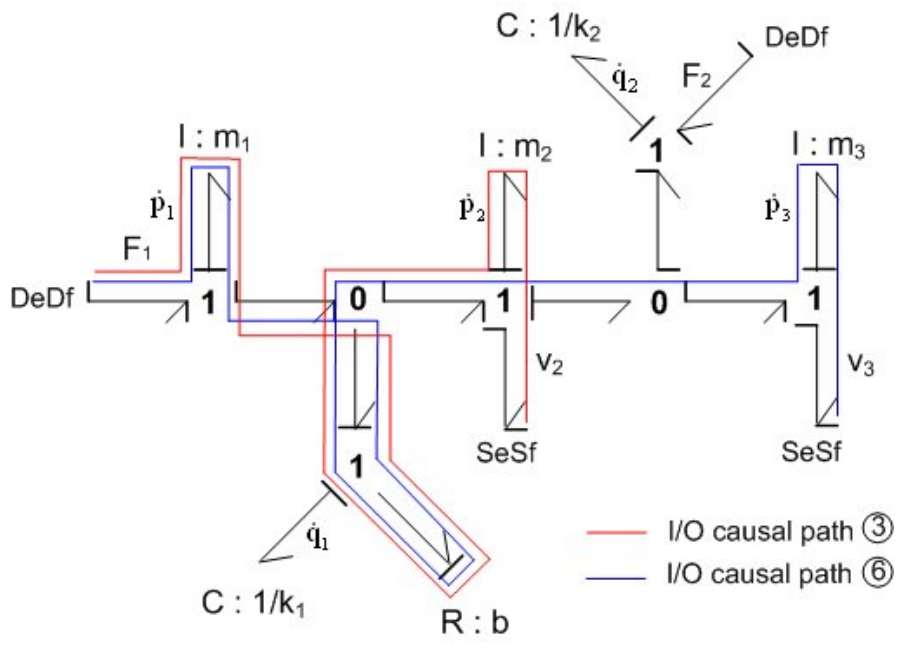

Fig. 15. Determination of essential orders in bicausal bond graph model.

the path (3) (resp. (6)) between $v_{2}$ (resp. $v_{3}$ ) and $F_{1}$ as shown in Fig. 15. So $n_{1 e}=-\omega_{3}\left(v_{2} \rightarrow F_{1}\right)=2$ and $n_{2 e}=-\omega_{6}\left(v_{3} \rightarrow F_{1}\right)=2$. 
These results are in agreement with those found using the state-space approach, Theorem 1.

\section{E. Interpretation and duality}

The causal and the bicausal procedures for the determination of relative orders and essential orders enable finding the same results as those found by the state-space approach. The causal procedure (procedure 7) enables determining the relative orders from the bond graph model in preferential integral causality by the analysis of the orders of the $\mathrm{I} / \mathrm{O}$ causal paths. The bicausal procedure (procedure 9) enables determining these orders from the bicausal bond graph model by the analysis of the orders of the I/O causal paths of the subsystems $\left(\mathbf{A}_{\mathbf{i n v}}, \overline{\mathbf{B}}_{\mathbf{i n v}_{\mathbf{i}}}(s), \mathbf{C}_{\mathbf{i n v}}, \overline{\mathbf{D}}_{\mathbf{i n v}_{\mathbf{i}}}(s)\right)$.

Also, procedure 8 enables determining the essential orders from the bond graph model in preferential integral causality by the analysis of the orders of the I/O causal paths of the subsystems $\left(\mathbf{A}, \mathbf{B}, \overline{\mathbf{C}}_{\mathbf{i}}\right)$. The procedure 10 enables determining these orders from the bicausal bond graph model by the analysis of the orders of I/O causal paths.

So, the causal procedure for the determination of the relative orders is the dual of the bicausal procedure for the determination of the essential orders and the causal procedure for the determination of the essential orders is the dual of the bicausal procedure for the determination of the relative orders. However attention has to be paid to the fact that whatever the bond graph representation (causal or bicausal), the output $y_{i}$ always refers to the same physical quantity. Talking about relative and essential orders on the bicausal model does not involve the outputs of the inverse model which are in fact the physical inputs of the system.

\section{CONCLUSiON}

In this paper, two bicausal procedures were given and demonstrated to graphically determine structural properties of linear time-invariant systems. The first procedure enables computing the infinite pole orders of the inverse model and the second procedure enables determining the relative orders from the bicausal bond graph model. To prove the latter procedure, a new state-space procedure to compute the relative order was given. In fact, in the literature and in the classical approaches (state-space approach, geometric approach, etc.), the relative order is defined from the direct model (before model inversion) because this order gives an idea of the necessary minimal time-differentiation order of each output $y_{i}$ in order to make appear at least one component of the input vector in the expression of $y_{i}^{\left(n_{i}^{\prime}\right)}$.

The causal procedure for the determination of the relative orders is simpler than the bicausal one, while the bicausal procedure for the determination of the essential orders is simpler than the causal one. So, the structural analysis of one bond graph model rather than another one depends on the aim of the analysis. The duality between the causal and bicausal procedures for the determination of relative orders and essential orders shows the duality between these invariants.

The proposed bond graph procedures represent a contribution to the structural analysis step of the methodology for sizing mechatronic systems according to energy and dynamic criteria. The main advantage of conducting structural analysis in the sizing methodology lies in the fact that the resulting diagnostic does not depend on parameter values or on the physical phenomena equations. Moreover, this approach offers the advantage to be entirely graphical and close to the physical meaning. The structural analysis step can help the engineer to write his specifications and then to correctly formulate his sizing problem (and this without running a simulation).

\section{REFERENCES}

[1] Dion J.M. , C. Commault, "Smith-Mcmillan factorisations at infinity of rational matrix functions and their control interpretation," Systems and Control Letters, vol. 1, pp. 312-320, 1982.

[2] Commault C. , J. Descusse, J.M. Dion, J.F. Lafay, M. Malabre, "New decoupling invariants: the essential orders," International Journal of Control, vol. 44, no. 3, pp. 689-700, 1986.

[3] Lafay J.F., P. Zagalak, A. Herrera, S. Icart, "Structural results about the interactor," in Proceedings of the IEEE Conference on Decision and Control, vol. 2, December 1990, pp. 1048-1049.

[4] Vardulakis A.I.G. , D.J.N. Limebeer, N. Karcanias, "Structure and Smith-MacMillan form of a rational matrix at infinity," International Journal of Control, vol. 35, pp. 701-725, 1982.

[5] Herrera A.N., J.F. Lafay, "New Results about Morgan's Problem," IEEE Transactions on Automatic Control, vol. 38, no. 12, pp. 1834-1838, December 1993.

[6] Commault C., J.M. Dion , "Structure at infinity of linear multivariable systems: a geometric approach," IEEE Transactions on Automatic Control, vol. AC-27, no. 3, pp. 693-696, Jun 1982.

[7] Descusse J. , J.F Lafay, M. Malabre , "Solution of the satic-state feedback decoupling problem for linear systems with two outputs," IEEE Transactions on Automatic Control, vol. AC-30, no. 9, pp. 914-918, 1985.

[8] - "Solution to Morgan problem," IEEE Transactions on Automatic Control, vol. 33, no. 8, pp. 732-739, 1988.

[9] Van Der Woude J. W. , "On the structure at infinity of a structured system," Linear algebra and its applications, vol. 148, pp. 145-169, 1991.

[10] Dion J.M., C. Commault, "Feedback decoupling of structured systems," IEEE Transactions on Automatic Control, vol. 38, no. 7, pp. 1132-1135, 1993.

[11] Karcanias N., E. Sagianos, E. Milonidis, "Structural identification: The computation of the generic McMillan degree," in Proceedings of the 44th IEEE Conference on Decision and Control, and the European Control Conference, CDC-ECC'05, Spain, December 2005, pp. 7222-7227.

[12] Karcanias N., E. Sagianos, E. Milonidis, "Structured transfer function matrices and integer matrices: The computation of the generic McMillan degree and infinite zero structure," International Jornal of Control, vol. 80, no. 9, pp. 1404-1420, September 2007.

[13] Reinschke K.J., Multivariable control: A Graph-theoretic approach, ser. Lecture notes in control and information sciences, Springer-Verlag, Ed., Berlain, 1988, no. ISBN 0-387-18899-1.

[14] Rahmani A., C. Sueur, G. Dauphin-Tanguy, "Formal determination of controllability/observability matrices for multivariable systems modelled by bond graph," in Proceedings of IMACS/SICE International Symposium of Robotics, Mechatronics and Manufacturing System, 1992, pp. 573-580.

[15] Wu S.T., K. Youcef-Toumi, “On Relative Degrees and Zero Dynamics From Physical System Modeling," Journal of Dynamic Systems, Measurement, and Control, vol. 117, no. 2, pp. 205-217, 1995.

[16] Rahmani A., C. Sueur, G. Dauphin-Tanguy, "On the infinite structure of systems modelled by bond graph: feedback decoupling," in Proceedings of the IEEE International Conference on Systems, Man and Cybernetics, vol. 3, Beijing, China, October 1996, pp. 1617-1622. 
[17] Bertrand J.M, C. Sueur, G. Dauphin-Tanguy, "On the finite and infinite structure of bond-graph models," IEEE Conference on Computational Cybernetics and Simulation, vol. 3, pp. 2472-2477, october 1997.

[18] Ngwompo R.F. , S. Scavarda, D. Thomasset, "Structural inversibility and minimal inversion of multivariable linear systems - a bond graph approach," Simulation Councils Proceedings Series, vol. 29, no. 1, p. 109, 1997.

[19] Ngwompo R.F., S. Scavarda, "Dimensioning problems in system design using bicausal bond graphs," Simulation Practice and Theory, vol. 7, no. 5, pp. 577-587, December 1999.

[20] Ngwompo R.F., P.J. Gawthrop, "Bond graph-based simulation of nonlinear inverse systems using physical performance specifications," Journal of the Franklin Institute, vol. 336, no. 8, pp. 1225-1247, 1999.

[21] Ngwompo R.F., S. Scavarda, D. Thomasset, "Physical model-based inversion in control systems design using bond graph representation, Part 1: theory," Proceedings of ImechE Journal of Systems and Control Engineering, vol. 215, no. 12, pp. 95-103, 2001.

[22] Glumineau A., C.H. Moog, "Nonlinear Morgan's Problem: Case of $(p+$ 1) Inputs and $p$ Outputs," IEEE Transactions on Automatic Control, vol. 37, no. 7, pp. 1067-1072, 1992.

[23] Birkett S.H. , P.H. Roe, "Mathematical foundations of bond graphs. III Matroid theory," Journal of Franklin Institute, vol. 327, pp. 87-108, 1990.

[24] Lichiardopol S., C. Sueur, "Duality of linear systems: a bond graph perspective," in 5th Mathmod Vienna, IMACS symposium on mathematical modeling, Vienna, February 2006.

[25] — "Duality in system analysis for bond graph models," Journal of Franklin Institute, 2009.

[26] Rahmani A., "Etude structurelle des systèmes linéaires par l'approche bond graph," Ph.D. dissertation, Université des Sciences et Technologie de Lille, France, 1993.

[27] Yamada T., T. Saga, "A sufficient condition for structural decouplability of linear non square systems," IEEE Transactions on Automatic Control, vol. 30, no. 9, pp. 918-921, September 1985.

[28] Jardin A., M. El Feki, W. Marquis-Favre, D. Thomasset, E. Bideaux, "Use of structural analysis in a bond graph-based methodology for sizing mechatronic systems," Le Grand Bornand, France, may 2008.

[29] Ngwompo R.F, E. Bideaux , S. Scavarda, "On the role of power lines and causal paths in bond graph based model inversion," Proceedings of International Conference on Bond Graph Modelling and Simulation: ICGBM'05, vol. 37(1), pp. 5-10, 2005.

[30] Dauphin-Tanguy G., Les bond graphs. Hermès Sciences, Paris, 2000.

[31] Jardin A., "Contribution à une méthodologie de dimensionnement des systèmes mécatroniques : analyse structurelle et couplage à l'optimisation dynamique," Ph.D. dissertation, Institut National des Sciences Appliquées de Lyon, 2010.

[32] Murota K., Matrices and matroids for systems analysis, ser. Algorithms and Combinatorics. Hardcover, 2000, vol. 20.

[33] Sueur C. , G. Dauphin-Tanguy, "Poles and zeros of multivariable linear systems: a bond graph approach," in Bond Graph for Engineers, P.C. Breedveld, G. Dauphin-Tanguy, Ed. Elsevier Science Publishers B.V., 1992, pp. 211-228.

[34] Karnopp D.C. , D.L. Margolis, R.C. Rosenberg, System Dynamics: A Unified Approach, J. Wiley, Ed., New York, 1975.

[35] Brockett R.W, "Poles, Zeros, and Feedback: State Space Interpretation," IEEE Transactions on Automatic Control, vol. AC-10, pp. 129-135, 1965.

[36] Descusse J., J.M. Dion, "On the Structure at Infinity of Linear Square Decoupled Systems," IEEE Transactions on Automatic Control, vol. AC27, no. 4, pp. 971-974, 1982.

[37] Commault C. , J.M. Dion, J. Torres, "Invariant spaces at infinity of linear systems application to block decoupling," IEEE Transactions on Automatic Control, vol. 35, no. 5, pp. 618-623, May 1990.

[38] El Feki M., Jardin A., Marquis-Favre W., Bideaux E., Thomasset D., "Determination of essential orders from a bond graph model," Submitted to Journal of Dynamic Systems, Measurement and Control, 2010.

[39] El Feki M. , M. Di Loreto, E. Bideaux, D. Thomasset, W. Marquis-Favre, "On the role of essential orders on feedback decoupling and model inversion: bond graph approach," in Proceedings of the 22th European Conference on Modelling and Simulation, Nicosia, Cyprus, june 2008. 